TRANSACTIONS OF THE

AMERICAN MATHEMATICAL SOCIETY

Volume 354, Number 6, Pages 2495-2520

S 0002-9947(02)02954- 9

Article electronically published on February 4, 2002

\title{
ALGEBRAIC AND SPECTRAL PROPERTIES OF DUAL TOEPLITZ OPERATORS
}

\author{
KAREL STROETHOFF AND DECHAO ZHENG
}

\begin{abstract}
Dual Toeplitz operators on the orthogonal complement of the Bergman space are defined to be multiplication operators followed by projection onto the orthogonal complement. In this paper we study algebraic and spectral properties of dual Toeplitz operators.
\end{abstract}

\section{INTRODUCTION}

The Bergman space $L_{a}^{2}$ is the Hilbert space of analytic functions on the unit disk $\mathbb{D}$ that are square integrable with respect to normalized area measure $d A$. We write $P$ for the orthogonal projection of $L^{2}(\mathbb{D}, d A)$ onto its closed linear subspace $L_{a}^{2}$. For a bounded measurable function $f$ on $\mathbb{D}$ the Toeplitz operator with symbol $f$ is the operator $T_{f}$ on $L_{a}^{2}$ defined by $T_{f} h=P(f h)$, for $h \in L_{a}^{2}$. We define the dual Toeplitz operator $S_{f}$ to be the operator on $\left(L_{a}^{2}\right)^{\perp}$ given by $S_{f} u=Q(f u)$, for $u \in\left(L_{a}^{2}\right)^{\perp}$, where $Q=I-P$ is the orthogonal projection of $L^{2}(\mathbb{D}, d A)$ onto $\left(L_{a}^{2}\right)^{\perp}$. The orthogonal complement of the Hardy space in $L^{2}(\partial \mathbb{D})$ is equal to $\overline{z H^{2}}$, so that a Toeplitz operator on $H^{2}$ is anti-unitarily equivalent to multiplication on $\left(H^{2}\right)^{\perp}$ followed by projection onto $\left(H^{2}\right)^{\perp}$. In the Bergman space setting this is no longer the case, since the orthogonal complement $\left(L_{a}^{2}\right)^{\perp}$ of $L_{a}^{2}$ in $L^{2}(\mathbb{D}, d A)$ is much larger than $\overline{z L_{a}^{2}}$.

Although dual Toeplitz operators differ in many ways from Toeplitz operators, they do have some of the same properties. The purpose of this paper is to study some algebraic and spectral properties of dual Toeplitz operators and to study to what extent these properties parallel those of Toeplitz operators on the Hardy space. Our results for dual Toeplitz operators may offer some insight into the study of similar questions for Toeplitz operators on the Bergman space.

In the setting of the classical Hardy space $H^{2}$, algebraic and spectral properties of Toeplitz operators were studied in [6], [9, 12], [16], 18] and [23]. In particular, Brown and Halmos [6] characterized commuting Toeplitz operators on $H^{2}$. Axler and Čučković $\left[3\right.$ characterized commutativity of Toeplitz operators on $L_{a}^{2}$ with bounded harmonic symbols. In Section 2 we will describe when two dual Toeplitz operators commute. Unlike the result of Axler and Čučković [3, our commuta-

Received by the editors March 10, 2000 and, in revised form, September 3, 2001.

2000 Mathematics Subject Classification. Primary 47B35, 47B47.

The second author was supported in part by the National Science Foundation and the University Research Council of Vanderbilt University. 
tivity result holds for general symbols. Brown and Halmos [6] showed that the product of two Toeplitz operators on the Hardy space can only be zero if one of the factors is zero. In Section 3 we will prove that dual Toeplitz operators have this same property. Two operators are called essentially commuting if their commutator is compact. In [19] the first author characterized essentially commuting Toeplitz operators on $L_{a}^{2}$ with harmonic symbols. A characterization of essentially commuting Toeplitz operators on the Hardy space has recently been obtained by Gorkin and the second author [11]. We will give a genenal characterization for essentially commuting dual Toeplitz operators in Section 4. In Section 5 we will give localized conditions for essential commutativity of pairs of dual Toeplitz operators whose symbols are continuous on the maximal ideal space of the algebra of bounded analytic functions on the unit disk. Analogously to McDonald and Sundberg's 15 description for the commutator ideal of the Toeplitz algebra, we will describe the commutator ideal of the $C^{*}$-algebra generated by all analytic dual Toeplitz operators in Section 6.

Brown and Halmos [6] showed that the only compact Toeplitz operator on the Hardy space is the zero operator and a Toeplitz operator is bounded on the Hardy space if and only if its symbol is bounded. This is easily seen to be false for Toeplitz operators on the Bergman space. A complete characterization of compact Toeplitz operators on the Bergman space via the Berezin transform has recently been obtained by Axler and the second author [5]. On the Bergman space, there are unbounded symbols that induce bounded Toeplitz operators. In Section 7 we prove that the only compact dual Toeplitz operator is the zero operator, and that a densely defined dual Toeplitz operator with square-integrable symbol is bounded if and only if its symbol is essentially bounded.

The symbol map on the Toeplitz algebra in the Hardy space has been an important tool in the study of Fredholm properties of Toeplitz operators and the structure of the Toeplitz algebra (see [9], Chapter 7). Analogous to the symbol map in the classical Hardy space setting, in Section 8 we construct a symbol map on the dual Toeplitz algebra, the algebra generated by all bounded dual Toeplitz operators. We establish structure theorems for the dual Toeplitz algebra and the $C^{*}$-subalgebra generated by the dual Toeplitz operators with symbols continuous on the closed unit disk. As an application of our symbol map we obtain a necessary condition on symbols of a finite number of dual Toeplitz operators whose product is the zero operator. For bounded harmonic functions on the unit disk we prove that the product of the associated dual Toeplitz operators can only be zero if one of the factors is the zero operator.

In the final section of the paper we discuss spectral properties of dual Toeplitz operators. We prove a spectral inclusion theorem, completely analogous to the spectral inclusion theorem of Hartman and Wintner [12] for Toeplitz operators on the Hardy space. Widom [23] proved that the spectrum of a Toeplitz operator on the Hardy space is connected, and Douglas 9 proved that also the essential spectrum is connected. We give examples to show that in general the spectrum and essential spectrum of a dual Toeplitz operator can be disconnected. For some classes of special symbols we establish connectedness of both the spectrum and essential spectrum of dual Toeplitz operators with these symbols.

Acknowledgement. We thank the referee for several comments that improved the paper, including a simplification of our proof of Theorem 9.12. 


\section{Commuting Dual Toeplitz Operators}

The following elementary algebraic properties of dual Toeplitz operators are easily verified:

$$
S_{f}^{*}=S_{\bar{f}},
$$

and

$$
S_{\alpha f+\beta g}=\alpha S_{f}+\beta S_{g},
$$

for bounded measurable functions $f$ and $g$ on $\mathbb{D}$, and $\alpha, \beta \in \mathbb{C}$. Both Toeplitz and dual Toeplitz operators are closely related to Hankel operators. For a bounded measurable function $f$ on $\mathbb{D}$ the Hankel operator $H_{f}$ is the operator $L_{a}^{2} \rightarrow\left(L_{a}^{2}\right)^{\perp}$ defined by $H_{f} h=Q(f h)$, for $h \in L_{a}^{2}$. Toeplitz operators on the Bergman space are related to Hankel operators by the following algebraic relation:

$$
T_{f g}=T_{f} T_{g}+H_{f}^{*} H_{g} .
$$

Analogously, for dual Toeplitz operators we have

$$
S_{f g}=S_{f} S_{g}+H_{f} H_{\bar{g}}^{*}
$$

The Hankel operator of an analytic symbol is the zero operator. Consequently, if $\varphi$ is a bounded analytic function on $\mathbb{D}$ and $\psi$ is a bounded measurable function on $\mathbb{D}$, then

$$
S_{\varphi} S_{\psi}=S_{\psi \varphi} \text { and } S_{\psi} S_{\bar{\varphi}}=S_{\psi \bar{\varphi}} .
$$

It follows from (2.2) that the dual Toeplitz operators $S_{f}$ and $S_{g}$ commute in case both $f$ and $g$ are analytic and in case both $f$ and $g$ are conjugate analytic. Clearly, the operators $S_{f}$ and $S_{g}$ commute also if a nontrivial linear combination of $f$ and $g$ is constant. The following theorem states that two dual Toeplitz operators commute only in these trivial cases. This result is completely analogous to the characterization of commuting Toeplitz operators on the Hardy space obtained by Brown and Halmos [6]. Unlike Axler and Cučković's [3] result for commutativity of Toeplitz operators on the Bergman space, we do not require the symbols to be harmonic.

Theorem 2.3. Let $f$ and $g$ be bounded measurable functions on $\mathbb{D}$. Then: $S_{f}$ and $S_{g}$ commute if and only if one of the following conditions holds:

(i) both $f$ and $g$ are analytic;

(ii) both $\bar{f}$ and $\bar{g}$ are analytic;

(iii) there are constants $\alpha$ and $\beta$, not both zero, such that $\alpha f+\beta g$ is constant.

Before we prove this theorem we discuss some preliminaries which will also be needed in the following sections.

The Bergman space $L_{a}^{2}$ has reproducing kernels $K_{w}$ given by

$$
K_{w}(z)=\frac{1}{(1-\bar{w} z)^{2}},
$$

for $z, w \in \mathbb{D}$ : for every $h \in L_{a}^{2}$ we have $\left\langle h, K_{w}\right\rangle=h(w)$, for all $w \in \mathbb{D}$. In particular, $\left\|K_{w}\right\|_{2}=\left\langle K_{w}, K_{w}\right\rangle^{1 / 2}=\left(1-|w|^{2}\right)^{-1}$. The functions

$$
k_{w}(z)=\frac{1-|w|^{2}}{(1-\bar{w} z)^{2}}
$$

are the normalized reproducing kernels for $L_{a}^{2}$. 
For $w \in \mathbb{D}$, the fractional linear transformation $\varphi_{w}$, defined by

$$
\varphi_{w}(z)=\frac{w-z}{1-\bar{w} z}
$$

for $z \in \mathbb{D}$, is an automorphism of the unit disk; in fact, $\varphi_{w}^{-1}=\varphi_{w}$.

For a linear operator $T$ on $\left(L_{a}^{2}\right)^{\perp}$ and $w \in \mathbb{D}$ we define the operator $\mathcal{S}_{w}(T)$ by

$$
\mathcal{S}_{w}(T)=T-S_{\varphi_{w}} T S_{\bar{\varphi}_{w}}
$$

Note that

$$
\mathcal{S}_{w}^{2}(T)=\mathcal{S}_{w}\left(\mathcal{S}_{w}(T)\right)=T-2 S_{\varphi_{w}} T S_{\bar{\varphi}_{w}}+S_{\varphi_{w}}^{2} T S_{\bar{\varphi}_{w}}^{2} .
$$

To get necessary conditions on dual Toeplitz operators with certain algebraic properties we will make use of rank one operators generated by the normalized reproducing kernels of the Bergman space. For $f, g \in L^{2}(\mathbb{D}, d A)$, define the rank one operator $f \otimes g$ by

$$
(f \otimes g) h=\langle h, g\rangle f,
$$

for $h \in L^{2}(\mathbb{D}, d A)$. It is easily shown that the norm of $f \otimes g$ is $\|f\|_{2}\|g\|_{2}$.

It follows from (2.1) that the commutator $\left[S_{f}, S_{g}\right]=S_{f} S_{g}-S_{g} S_{f}$ is given by

$$
\left[S_{f}, S_{g}\right]=H_{g} H_{f}^{*}-H_{f} H_{\bar{g}}^{*}
$$

From the proof of Proposition 4.8 of [22],

$$
\left(H_{f} k_{w}\right) \otimes\left(H_{\bar{g}} k_{w}\right)=H_{f}\left(k_{w} \otimes k_{w}\right) H_{\bar{g}}^{*}=\mathcal{S}_{w}^{2}\left(H_{f} H_{\bar{g}}^{*}\right) .
$$

Also,

$$
\left(H_{g} k_{w}\right) \otimes\left(H_{\bar{f}} k_{w}\right)=\mathcal{S}_{w}^{2}\left(H_{g} H_{\bar{f}}^{*}\right) .
$$

Using (2.4) it follows that

$$
\left(H_{g} k_{w}\right) \otimes\left(H_{\bar{f}} k_{w}\right)-\left(H_{f} k_{w}\right) \otimes\left(H_{\bar{g}} k_{w}\right)=\mathcal{S}_{w}^{2}\left(\left[S_{f}, S_{g}\right]\right) .
$$

We are now ready to prove Theorem 2.3

Proof of Theorem 2.3. It suffices to show the necessity of one of conditions (i), (ii) and (iii) in case $S_{f}$ and $S_{g}$ commute. If $S_{f}$ and $S_{g}$ commute, then it follows from (2.6) that $\left(H_{f} k_{w}\right) \otimes\left(H_{\bar{g}} k_{w}\right)=\left(H_{g} k_{w}\right) \otimes\left(H_{\bar{f}} k_{w}\right)$, for all $w \in \mathbb{D}$. Note that $k_{0} \equiv 1$, thus

$$
\left(H_{f} 1\right) \otimes\left(H_{\bar{g}} 1\right)=\left(H_{g} 1\right) \otimes\left(H_{\bar{f}} 1\right)
$$

that is,

$$
\left\langle u, H_{\bar{g}} 1\right\rangle H_{f} 1=\left\langle u, H_{\bar{f}} 1\right\rangle H_{g} 1,
$$

for all $u \in\left(L_{a}^{2}\right)^{\perp}$. If $H_{\bar{f}} 1 \neq 0$ and $H_{f} 1 \neq 0$, then there exists a complex number $\lambda$ such that $H_{g} 1=\lambda H_{f} 1$ and $H_{\bar{g}} 1=\bar{\lambda} H_{\bar{f}} 1$. Then $Q(g-\lambda f)=0$, so that the function $g-\lambda f$ is analytic. Also $Q(\bar{g}-\bar{\lambda} \bar{f})=0$, so that $g-\lambda f$ is also co-analytic. Thus $g-\lambda f$ is constant. If $H_{\bar{f}} 1=0$, then $f$ is co-analytic, and $H_{f} 1=0$ or $H_{\bar{g}} 1=0$, that is, $f$ is also analytic (in which case $f$ is constant) or $g$ is also co-analytic. If $H_{f} 1=0$, then $f$ is analytic, and $H_{g} 1=0$ or $H_{\bar{f}} 1=0$, that is, $g$ is analytic or $f$ is also co-analytic (in which case $f$ is constant). 
The above proof does not work for Toeplitz operators, because there is no canonical transformation of Hankel products $H_{\bar{f}}^{*} H_{g}$ into rank-one operators such as the transformation used to obtain equation (2.5).

Theorem 2.3 has the following consequence.

Corollary 2.7. Let $f$ be a bounded measurable function on $\mathbb{D}$. Then the dual Toeplitz operator $S_{f}$ is normal if and only if the range of $f$ lies on a line.

Proof. The dual Toeplitz operator $S_{f}$ is normal if and only if $S_{f}$ and $S_{f}^{*}=S_{\bar{f}}$ commute. By Theorem 2.3 this is the case if and only if there are constants $\alpha$ and $\beta$, not both zero, such that $\alpha f+\beta \bar{f}$ is constant.

\section{Zero Divisors of Dual Toeplitz Operators}

Brown and Halmos proved that the product of two Toeplitz operators on the Hardy space can only be zero if one of the Toeplitz operators is zero. Whether the analogous statement is true for Toeplitz operators on the Bergman space is still an open question, even if the symbols are restricted to harmonic functions. Ahern and Cučković [1] have recently obtained results in support of the conjecture that the above question has an affirmative answer for harmonic symbols. In this section we will prove the analogous result for dual Toeplitz operators.

We have the following theorem, analogous to Theorem 8 of Brown and Halmos [6]:

Theorem 3.1. Let $f$ and $g$ be bounded measurable functions on $\mathbb{D}$. Then: $S_{f} S_{g}$ is a dual Toeplitz operator if and only if $f$ is analytic on $\mathbb{D}$ or $g$ is co-analytic on $\mathbb{D}$, in which case $S_{f} S_{g}=S_{f g}$.

Proof. The sufficiency follows immediately from (2.2). To prove the necessity, suppose that $S_{f} S_{g}=S_{h}$, where $h$ is a bounded measurable function on $\mathbb{D}$. Then it follows from (2.1) that

$$
S_{f g-h}=H_{f} H_{\bar{g}}^{*}
$$

Using (2.2) we see that

$$
\mathcal{S}_{w}\left(S_{f g-h}\right)=S_{f g-h}-S_{\varphi_{w}} S_{f g-h} S_{\bar{\varphi}_{w}}=S_{\left(1-\left|\varphi_{w}\right|^{2}\right)(f g-h)},
$$

hence

It follows that

$$
\mathcal{S}_{w}^{2}\left(S_{f g-h}\right)=S_{\left(1-\left|\varphi_{w}\right|^{2}\right)^{2}(f g-h)}
$$

$$
S_{\left(1-\left|\varphi_{w}\right|^{2}\right)^{2}(f g-h)}=\mathcal{S}_{w}^{2}\left(S_{f g-h}\right)=\mathcal{S}_{w}^{2}\left(H_{f} H_{\bar{g}}^{*}\right)=\left(H_{f} k_{w}\right) \otimes\left(H_{\bar{g}} k_{w}\right),
$$

for all $w \in \mathbb{D}$. In particular,

$$
S_{\left(1-|z|^{2}\right)^{2}(f g-h)}=\left(H_{f} 1\right) \otimes\left(H_{\bar{g}} 1\right) .
$$

Using that the range of $S_{\left(1-|z|^{2}\right)^{2}(f g-h)}$ is at most one-dimensional, it is easy to see that there exist complex numbers $a$ and $b$, not both zero, such that

$$
S_{\left(1-|z|^{2}\right)^{2}(f g-h)}\left(a \bar{z}+b \bar{z}^{2}\right)=0 .
$$

This implies that the function $\varphi=\left(1-|z|^{2}\right)^{2}(f g-h)\left(a \bar{z}+b \bar{z}^{2}\right)$ is in $L_{a}^{2}$. Since $\varphi(z) \rightarrow 0$ as $|z| \rightarrow 1^{-}$, we must have $\varphi(z)=0$, for all $z \in \mathbb{D}$. Thus $f(z) g(z)=$ $h(z)$ for all $z \in \mathbb{D}$, with the exception of at most two points. It follows that $\left(H_{f} 1\right) \otimes\left(H_{\bar{g}} 1\right)=0$, thus $\left\|H_{f} 1\right\|_{2}\left\|H_{\bar{g}} 1\right\|_{2}=0$. If $H_{f} 1=0$, then $f$ is analytic on $\mathbb{D}$; if $H_{\bar{g}} 1=0$, then $\bar{g}$ is analytic on $\mathbb{D}$. 
The following corollary states that dual Toeplitz operators have no zero-divisors.

Corollary 3.2. The product of two dual Toeplitz operators can only be zero if one of the dual Toeplitz operators is zero.

Proof. If $S_{f} S_{g}=0$, then $S_{f} S_{g}=S_{h}$, where $h$ is the zero function. From the proof of Theorem [3.1] we see that $f g=0$ on $\mathbb{D}$. This implies that $f=0$ almost everywhere or $g=0$ almost everywhere, thus $S_{f}=0$ or $S_{g}=0$.

Corollary 3.3. A dual Toeplitz operator $S_{f}$ is an isometry if and only if $f$ is constant of modulus 1 .

Proof. By Theorem $3.1, S_{\bar{f}} S_{f}=I=S_{1}$ only if $f$ is analytic, in which case $S_{\bar{f}} S_{f}=$ $S_{|f|^{2}}$. Thus $|f|^{2}=1$ on $\mathbb{D}$, which is only possible if $f$ is constant.

Corollary 3.4. The only idempotent dual Toeplitz operators are 0 and I.

Proof. If $S_{f}^{2}=S_{f}$, then $S_{f} S_{1-f}=0$, and by Corollary [3.2, $S_{f}=0$, or $S_{1-f}=0$ (in which case $S_{f}=S_{1}=I$ ).

\section{Essentially Commuting Dual Toeplitz Operators}

We have the following result for compactness of the commutator of a pair of dual Toeplitz operators. In the next section we will use this theorem to show that for nice symbols $f$ and $g$ the dual Toeplitz operators $S_{f}$ and $S_{g}$ are essentially commuting if and only if the conditions for commutativity hold locally. This will be made precise in the next section.

Theorem 4.1. Let $f$ and $g$ be bounded measurable functions on $\mathbb{D}$. Then the commutator $\left[S_{f}, S_{g}\right]$ is compact if and only if

$$
\left\|\left(H_{g} k_{w}\right) \otimes\left(H_{\bar{f}} k_{w}\right)-\left(H_{f} k_{w}\right) \otimes\left(H_{\bar{g}} k_{w}\right)\right\| \rightarrow 0,
$$

as $|w| \rightarrow 1^{-}$.

We need the following lemma from [22].

Lemma 4.2. If $T$ is a compact operator on $\left(L_{a}^{2}\right)^{\perp}$, then $\left\|\mathcal{S}_{w}(T)\right\| \rightarrow 0$ as $|w| \rightarrow 1^{-}$.

Note that $\left\|\mathcal{S}_{w}(T)\right\| \leq 2\|T\|$. Thus $\left\|\mathcal{S}_{w}^{2}(T)\right\| \leq 2\left\|\mathcal{S}_{w}(T)\right\|$, so if $T$ is a compact operator on $\left(L_{a}^{2}\right)^{\perp}$, then by Lemma 4.2 also $\left\|\mathcal{S}_{w}^{2}(T)\right\| \rightarrow 0$ as $|w| \rightarrow 1^{-}$. Thus, if $\left[S_{f}, S_{g}\right]$ is compact, then $\left\|\mathcal{S}_{w}^{2}\left(\left[S_{f}, S_{g}\right]\right)\right\| \rightarrow 0$ as $|w| \rightarrow 1^{-}$, and the necessity of the condition in Theorem 4.1 follows using (2.6).

To prove the sufficiency of the condition in Theorem 4.1 we will make use of the following lemmas.

Lemma 4.3. If $u_{1}, u_{2}, v_{1}, v_{2}$ are vectors in a Hilbert space $\mathcal{H}$ with $u_{1} \perp u_{2}$, then

$$
\frac{1}{2}\left(\left\|u_{1}\right\|\left\|v_{1}\right\|+\left\|u_{2}\right\|\left\|v_{2}\right\|\right) \leq\left\|u_{1} \otimes v_{1}+u_{2} \otimes v_{2}\right\| \leq\left\|u_{1}\right\|\left\|v_{1}\right\|+\left\|u_{2}\right\|\left\|v_{2}\right\| .
$$

Proof. Putting $S=u_{1} \otimes v_{1}+u_{2} \otimes v_{2}$, we have

$$
\|S x\|^{2}=\left|\left\langle x, v_{1}\right\rangle\right|^{2}\left\|u_{1}\right\|^{2}+\left|\left\langle x, v_{2}\right\rangle\right|^{2}\left\|u_{2}\right\|^{2} \leq\|x\|^{2}\left\|v_{1}\right\|^{2}\left\|u_{1}\right\|^{2}+\|x\|^{2}\left\|v_{2}\right\|^{2}\left\|u_{2}\right\|^{2},
$$

for all $x \in \mathcal{H}$, and therefore $\|S\|^{2} \leq\left\|u_{1}\right\|^{2}\left\|v_{1}\right\|^{2}+\left\|u_{2}\right\|^{2}\left\|v_{2}\right\|^{2}$. Clearly we also have $\left\|u_{1}\right\|^{2}\left\|v_{1}\right\|^{2} \leq\|S\|^{2}$ and $\left\|u_{2}\right\|^{2}\left\|v_{2}\right\|^{2} \leq\|S\|^{2}$. Hence $\frac{1}{2}\left(\left\|u_{1}\right\|^{2}\left\|v_{1}\right\|^{2}+\left\|u_{2}\right\|^{2}\left\|v_{2}\right\|^{2}\right) \leq$ $\|S\|^{2}$. The stated result follows using the inequalities $\left(s^{2}+t^{2}\right) \leq(s+t)^{2} \leq 2\left(s^{2}+t^{2}\right)$, for $s, t \geq 0$. 
Before we state and prove another lemma, we introduce more notation. For $w \in \mathbb{D}$ we use $F_{w}$ to denote the following finite rank operator on $\left(L_{a}^{2}\right)^{\perp}$ :

$$
F_{w}=H_{f} k_{w} \otimes H_{\bar{g}} k_{w}-H_{g} k_{w} \otimes H_{\bar{f}} k_{w} .
$$

Lemma 4.4. Let $f$ and $g$ be bounded measurable functions on $\mathbb{D}$. If neither $f$ nor $g$ is analytic on $\mathbb{D}$, then

$$
\left\|F_{w}\right\| \leq\left\|H_{f} k_{w}\right\|_{2}\left\|H_{\overline{g-\lambda f}} k_{w}\right\|_{2}+\left\|H_{\bar{f}} k_{w}\right\|_{2}\left\|H_{g-\lambda f} k_{w}\right\|_{2} \leq 2\left\|F_{w}\right\|
$$

and

$$
\left\|F_{w}\right\| \leq\left\|H_{f-\mu g} k_{w}\right\|_{2}\left\|H_{\bar{g}} k_{w}\right\|_{2}+\left\|H_{\overline{f-\mu g}} k_{w}\right\|_{2}\left\|H_{g} k_{w}\right\|_{2} \leq 2\left\|F_{w}\right\|
$$

where

$$
\lambda=\frac{\left\langle H_{g} k_{w}, H_{f} k_{w}\right\rangle}{\left\|H_{f} k_{w}\right\|_{2}^{2}} \text { and } \mu=\frac{\left\langle H_{f} k_{w}, H_{g} k_{w}\right\rangle}{\left\|H_{g} k_{w}\right\|_{2}^{2}} .
$$

Proof. We have $H_{g-\lambda f} k_{w} \perp H_{f} k_{w}$ and

$$
F_{w}=\left(H_{f} k_{w}\right) \otimes\left(H_{\overline{g-\lambda f}} k_{w}\right)-\left(H_{g-\lambda f} k_{w}\right) \otimes\left(H_{\bar{f}} k_{w}\right) .
$$

The first pair of inequalities follows using Lemma 4.3. The second pair of inequalities is proved similarly.

In the proof of Theorem 4.1 we need some results from [22]. The following inner product formula is proved in [22]:

$$
\begin{aligned}
\int_{\mathbb{D}} F(z) \overline{G(z)} d A(z)=3 \int_{\mathbb{D}}\left(1-|z|^{2}\right)^{2} F(z) \overline{G(z)} d A(z) \\
+\frac{1}{2} \int_{\mathbb{D}}\left(1-|z|^{2}\right)^{2} F^{\prime}(z) \overline{G^{\prime}(z)} d A(z) \\
+\frac{1}{3} \int_{\mathbb{D}}\left(1-|z|^{2}\right)^{3} F^{\prime}(z) \overline{G^{\prime}(z)} d A(z)
\end{aligned}
$$

for $F$ and $G$ in $L_{a}^{2}$. If $h$ is a bounded measurable function on $\mathbb{D}, u \in\left(L_{a}^{2}\right)^{\perp}$, and $\varepsilon>0$, then in 22 it is shown that

$$
\left(1-|z|^{2}\right)\left|\left(H_{h}^{*} u\right)(z)\right| \leq\left\|h \circ \varphi_{z}-P\left(h \circ \varphi_{z}\right)\right\|_{2}\|u\|_{2},
$$

and

$$
\left(1-|z|^{2}\right)\left|\left(H_{h}^{*} u\right)^{\prime}(z)\right| \leq 4\left\|h \circ \varphi_{z}-P\left(h \circ \varphi_{z}\right)\right\|_{2+\varepsilon} P_{0}\left[|u|^{\delta}\right](z)^{1 / \delta},
$$

for every $z \in \mathbb{D}$, where $\delta=(2+\varepsilon) /(1+\varepsilon)$ and $P_{0}$ denotes the integral operator on $L^{2}(\mathbb{D}, d A)$ with kernel $1 /|1-\bar{w} z|^{2}$. It is well-known that $P_{0}$ is $L^{p}$-bounded for every $1<p<\infty$ (see [2] or [24]).

Proof of Theorem 4.1. Let $0<s<1$. Using the inner product formula (4.5), it is easily seen that there exists a compact operator $K_{s}$ on $\left(L_{a}^{2}\right)^{\perp}$ such that

$$
\left\langle\left(\left[S_{f}, S_{g}\right]-K_{s}\right) u, v\right\rangle=I_{s}+I I_{s}+I I I_{s}
$$

for $u, v \in\left(L_{a}^{2}\right)^{\perp}$, where

$$
\begin{gathered}
I_{s}=3 \int_{s<|z|<1}\left(1-|z|^{2}\right)^{2}\left\{\left(H_{\bar{f}}^{*} u\right)(z) \overline{\left(H_{g}^{*} v\right)(z)}-\left(H_{\bar{g}}^{*} u\right)(z) \overline{\left(H_{f}^{*} v\right)(z)}\right\} d A(z), \\
I I_{s}=\frac{1}{2} \int_{s<|z|<1}\left(1-|z|^{2}\right)^{2}\left\{\left(H_{\bar{f}}^{*} u\right)^{\prime}(z) \overline{\left(H_{g}^{*} v\right)^{\prime}(z)}-\left(H_{\bar{g}}^{*} u\right)^{\prime}(z) \overline{\left(H_{f}^{*} v\right)^{\prime}(z)}\right\} d A(z),
\end{gathered}
$$


and

$$
I I I_{s}=\frac{1}{3} \int_{s<|z|<1}\left(1-|z|^{2}\right)^{3}\left\{\left(H_{\bar{f}}^{*} u\right)^{\prime}(z) \overline{\left(H_{g}^{*} v\right)^{\prime}(z)}-\left(H_{\bar{g}}^{*} u\right)^{\prime}(z) \overline{\left(H_{f}^{*} v\right)^{\prime}(z)}\right\} d A(z) .
$$

We will estimate each of $\left|I_{s}\right|,\left|I I_{s}\right|$ and $\left|I I I_{s}\right|$.

By (4.6) we have,

$$
\left(1-|z|^{2}\right)^{2}\left|\left(H_{\bar{f}}^{*} u\right)(z) \overline{\left(H_{g}^{*} v\right)(z)}-\left(H_{\bar{g}}^{*} u\right)(z) \overline{\left(H_{f}^{*} v\right)(z)}\right| \leq\|u\|_{2}\|v\|_{2} R(f, g, z),
$$

where

$$
R(f, g, z)=\left\|Q\left(f \circ \varphi_{z}\right)\right\|_{2}\left\|Q\left(\bar{g} \circ \varphi_{z}\right)\right\|_{2}+\left\|Q\left(\bar{f} \circ \varphi_{z}\right)\right\|_{2}\left\|Q\left(g \circ \varphi_{z}\right)\right\|_{2} .
$$

It is easily verified that

$$
\begin{aligned}
\left(H_{\bar{f}}^{*} u\right)(z) & \overline{\left(H_{g}^{*} v\right)(z)}-\left(H_{\bar{g}}^{*} u\right)(z) \overline{\left(H_{f}^{*} v\right)(z)} \\
& =\left(H_{\bar{f}}^{*} u\right)(z) \overline{\left(H_{g-\lambda f}^{*} v\right)(z)}-\left(H_{\bar{g}-\lambda f}^{*} u\right)(z) \overline{\left(H_{f}^{*} v\right)(z)},
\end{aligned}
$$

for all $z \in \mathbb{D}$ and $\lambda \in \mathbb{C}$; thus also

$$
\left(1-|z|^{2}\right)^{2}\left|\left(H_{\bar{f}}^{*} u\right)(z) \overline{\left(H_{g}^{*} v\right)(z)}-\left(H_{\bar{g}}^{*} u\right)(z) \overline{\left(H_{f}^{*} v\right)(z)}\right| \leq\|u\|_{2}\|v\|_{2} R(f, g-\lambda f, z),
$$

for all $z \in \mathbb{D}$ and $\lambda \in \mathbb{C}$. We have a similar inequality with $f$ replaced by $f-\mu g$, and conclude that

$$
\left(1-|z|^{2}\right)^{2}\left|\left(H_{\bar{f}}^{*} u\right)(z) \overline{\left(H_{g}^{*} v\right)(z)}-\left(H_{\bar{g}}^{*} u\right)(z) \overline{\left(H_{f}^{*} v\right)(z)}\right| \leq\|u\|_{2}\|v\|_{2} M(f, g, z),
$$

for all $z \in \mathbb{D}$, where

$$
M(f, g, z)=\min \left\{\min _{|\lambda| \leq 1} R(f, g-\lambda f, z), \min _{|\mu| \leq 1} R(f-\mu g, g, z)\right\} .
$$

It follows that

$$
\left|I_{s}\right| \leq 3\|u\|_{2}\|v\|_{2} \sup _{s<|z|<1} M(f, g, z) .
$$

Next we will obtain similar estimates for $\left|I I_{s}\right|$ and $\left|I I I_{s}\right|$. By (4.7) we have

$$
\begin{aligned}
\left(1-|z|^{2}\right)^{2} & \left|\left(H_{\bar{f}}^{*} u\right)^{\prime}(z) \overline{\left(H_{g}^{*} v\right)^{\prime}(z)}-\left(H_{\bar{g}}^{*} u\right)^{\prime}(z) \overline{\left(H_{f}^{*} v\right)^{\prime}(z)}\right| \\
& \leq 16 P_{0}\left[|u|^{\delta}\right](z)^{1 / \delta} P_{0}\left[|v|^{\delta}\right](z)^{1 / \delta} R_{\varepsilon}(f, g, z),
\end{aligned}
$$

where

$$
R_{\varepsilon}(f, g, z)=\left\|Q\left(f \circ \varphi_{z}\right)\right\|_{2+\varepsilon}\left\|Q\left(\bar{g} \circ \varphi_{z}\right)\right\|_{2+\varepsilon}+\left\|Q\left(\bar{f} \circ \varphi_{z}\right)\right\|_{2+\varepsilon}\left\|Q\left(g \circ \varphi_{z}\right)\right\|_{2+\varepsilon} .
$$

It follows from (4.8) that

$$
\left(H_{\bar{f}}^{*} u\right)^{\prime}(z) \overline{\left(H_{g}^{*} v\right)^{\prime}(z)}-\left(H_{\bar{g}}^{*} u\right)^{\prime}(z) \overline{\left(H_{f}^{*} v\right)^{\prime}(z)}
$$

is invariant if $g$ is replaced by $g-\lambda f$ or $f$ is replaced by $f-\mu g$. We obtain

$$
\begin{aligned}
\left(1-|z|^{2}\right)^{2} & \left|\left(H_{\bar{f}}^{*} u\right)^{\prime}(z) \overline{\left(H_{g}^{*} v\right)^{\prime}(z)}-\left(H_{\bar{g}}^{*} u\right)^{\prime}(z) \overline{\left(H_{f}^{*} v\right)^{\prime}(z)}\right| \\
& \leq 16 P_{0}\left[|u|^{\delta}\right](z)^{1 / \delta} P_{0}\left[|v|^{\delta}\right](z)^{1 / \delta} M_{\varepsilon}(f, g, z),
\end{aligned}
$$

where

$$
M_{\varepsilon}(f, g, z)=\min \left\{\min _{|\lambda| \leq 1} R_{\varepsilon}(f, g-\lambda f, z), \min _{|\mu| \leq 1} R_{\varepsilon}(f-\mu g, g, z)\right\} .
$$


It follows that

$$
\begin{aligned}
\left|I I_{s}\right| & \leq 8 \int_{s<|z|<1} P_{0}\left[|u|^{\delta}\right](z)^{1 / \delta} P_{0}\left[|v|^{\delta}\right](z)^{1 / \delta} M_{\varepsilon}(f, g, z) d A(z) \\
& \leq 8 \sup _{s<|z|<1} M_{\varepsilon}(f, g, z) \int_{\mathbb{D}} P_{0}\left[|u|^{\delta}\right](z)^{1 / \delta} P_{0}\left[|v|^{\delta}\right](z)^{1 / \delta} d A(z) .
\end{aligned}
$$

By the Cauchy-Schwarz inequality, and $L^{p}$-boundedness of the operator $P_{0}$ (for $p=2 / \delta>1)$,

$$
\begin{aligned}
\int_{\mathbb{D}} P_{0} & {\left[|u|^{\delta}\right](z)^{1 / \delta} P_{0}\left[|v|^{\delta}\right](z)^{1 / \delta} d A(z) } \\
& \leq\left(\int_{\mathbb{D}} P_{0}\left[|u|^{\delta}\right](z)^{2 / \delta} d A(z)\right)^{1 / 2}\left(\int_{\mathbb{D}} P_{0}\left[|v|^{\delta}\right](z)^{2 / \delta} d A(z)\right)^{1 / 2} \\
& \leq\left(C \int_{\mathbb{D}}\left[|u(z)|^{\delta}\right]^{2 / \delta} d A(z)\right)^{1 / 2}\left(C \int_{\mathbb{D}}\left[|v(z)|^{\delta}\right]^{2 / \delta} d A(z)\right)^{1 / 2} \\
& =C\|u\|_{2}\|v\|_{2}
\end{aligned}
$$

Thus

$$
\left|I I_{s}\right| \leq 8 C\|u\|_{2}\|v\|_{2} \sup _{s<|z|<1} M_{\varepsilon}(f, g, z) .
$$

Similarly we obtain the estimate

$$
\left|I I I_{s}\right| \leq 8 C\|u\|_{2}\|v\|_{2} \sup _{s<|z|<1} M_{\varepsilon}(f, g, z) .
$$

Since clearly $M(f, g, z) \leq M_{\varepsilon}(f, g, z)$ we can conclude that there exists a finite positive constant $C^{\prime}$ for which

$$
\left\|\left[S_{f}, S_{g}\right]-K_{s}\right\| \leq C^{\prime} \sup _{s<|z|<1} M_{\varepsilon}(f, g, z) .
$$

It remains to show that $M_{\varepsilon}(f, g, w) \rightarrow 0$ as $|w| \rightarrow 1^{-}$. This will follow from the following:

Claim. There is a constant $C$ such that $M_{\varepsilon}(f, g, w) \leq C\left\|F_{w}\right\|^{1 /(2+\varepsilon)}$, for all $w \in \mathbb{D}$.

To prove the claim, fix $w \in \mathbb{D}$. Suppose that $\left\|H_{f} k_{w}\right\|>0$ and $\left\|H_{g} k_{w}\right\|>0$. Let $\lambda$ and $\mu$ be as in Lemma 4.4 By the Cauchy-Schwarz inequality, $|\lambda||\mu|=\lambda \mu \leq 1$, thus $|\lambda| \leq 1$ or $|\mu| \leq 1$. Assume that $|\lambda| \leq 1$. Note that then

$$
M_{\varepsilon}(f, g, w) \leq R_{\varepsilon}(f, g-\lambda f, w) .
$$

By Lemma 4.4 $R(f, g-\lambda f, w) \leq 2\left\|F_{w}\right\|$.

If $h$ is a bounded measurable function on $\mathbb{D}$, using the Cauchy-Schwarz inequality we have

$$
\left\|Q\left(h \circ \varphi_{w}\right)\right\|_{2+\varepsilon} \leq\left\|Q\left(h \circ \varphi_{w}\right)\right\|_{2}^{1 /(2+\varepsilon)}\left\|Q\left(h \circ \varphi_{w}\right)\right\|_{2+2 \varepsilon}^{(1+\varepsilon) /(2+\varepsilon)} .
$$

Since the Bergman projection $P$ is $L^{2+2 \varepsilon}$-bounded, we can find a positive constant $M$ such that $\left\|Q\left(h \circ \varphi_{w}\right)\right\|_{2+2 \varepsilon} \leq M\|h\|_{\infty}$, for all $w \in \mathbb{D}$. It follows that

$$
\begin{aligned}
& \left\|Q\left(f \circ \varphi_{w}\right)\right\|_{2+\varepsilon}\left\|Q\left((\overline{g-\lambda f}) \circ \varphi_{w}\right)\right\|_{2+\varepsilon} \\
& \quad \leq M^{2 / \delta}\|f\|_{\infty}^{1 / \delta}\|g-\lambda f\|_{\infty}^{1 / \delta}\left\|Q\left(f \circ \varphi_{w}\right)\right\|_{2}^{1 /(2+\varepsilon)}\left\|Q\left((\overline{g-\lambda f}) \circ \varphi_{w}\right)\right\|_{2}^{1 /(2+\varepsilon)} \\
& \quad \leq M^{2 / \delta}\left(\|f\|_{\infty}+\|g\|_{\infty}\right)^{2 / \delta}\left\|Q\left(f \circ \varphi_{w}\right)\right\|_{2}^{1 /(2+\varepsilon)}\left\|Q\left((\overline{g-\lambda f}) \circ \varphi_{w}\right)\right\|_{2}^{1 /(2+\varepsilon)},
\end{aligned}
$$


for all $w \in \mathbb{D}$. We conclude that

$$
\begin{aligned}
R_{\varepsilon}(f, g-\lambda f, w) \leq & M^{2 / \delta}\left(\|f\|_{\infty}+\|g\|_{\infty}\right)^{2 / \delta} \\
\times\{ & \left(\left\|Q\left(f \circ \varphi_{w}\right)\right\|_{2}\left\|Q\left((\overline{g-\lambda f}) \circ \varphi_{w}\right)\right\|_{2}\right)^{1 /(2+\varepsilon)} \\
& \left.\quad+\left(\left\|Q\left(\bar{f} \circ \varphi_{w}\right)\right\|_{2}\left\|Q\left((g-\lambda f) \circ \varphi_{w}\right)\right\|_{2}\right)^{1 /(2+\varepsilon)}\right\},
\end{aligned}
$$

for all $w \in \mathbb{D}$. Applying the inequality $s^{1 / p}+t^{1 / p} \leq 2^{(p-1) / p}(s+t)^{1 / p}$ with $s=$ $\left.\left\|Q\left(f \circ \varphi_{w}\right)\right\|_{2} \| Q(\overline{g-\lambda f}) \circ \varphi_{w}\right)\left\|_{2}, t=\right\| Q\left(\bar{f} \circ \varphi_{w}\right)\left\|_{2}\right\| Q\left((g-\lambda f) \circ \varphi_{w}\right) \|_{2}$ and $p=2+\varepsilon$, we obtain

$$
R_{\varepsilon}(f, g-\lambda f, w) \leq 2^{1 / \delta} M^{2 / \delta}\left(\|f\|_{\infty}+\|g\|_{\infty}\right)^{2 / \delta} R(f, g-\lambda f, w)^{1 /(2+\varepsilon)},
$$

for all $w \in \mathbb{D}$. It follows that

$$
M_{\varepsilon}(f, g, w) \leq 2^{1 / \delta} M^{2 / \delta}\left(\|f\|_{\infty}+\|g\|_{\infty}\right)^{2 / \delta}\left\|F_{w}\right\|^{1 /(2+\varepsilon)},
$$

for all $w \in \mathbb{D}$, proving the claim.

In case $H_{f} k_{w}=0$, we have

$$
\left\|F_{w}\right\|=\left\|H_{g} k_{w} \otimes H_{\bar{f}} k_{w}\right\|=\left\|H_{g} k_{w}\right\|_{2}\left\|H_{\bar{f}} k_{w}\right\|_{2}=R(f, g, w),
$$

and, reasoning as before, the inequality follows. The case $H_{\bar{f}} k_{w}=0$ is treated similarly.

\section{Symbols Continuous on the Maximal Ideal Space}

Let $H^{\infty}$ denote the algebra of bounded analytic functions on $\mathbb{D}$. The maximal ideal space of $H^{\infty}$, denoted by $\mathcal{M}$, is the set of all multiplicative linear functionals on $H^{\infty}$. Endowed with the weak-star topology it inherits as a subspace of the dual of $H^{\infty}$, the maximal ideal space $\mathcal{M}$ is a compact Hausdorff space. Using the Gelfand transform we think of $H^{\infty}$ as a subalgebra of $C(\mathcal{M})$, the algebra of continuous complex-valued functions on $\mathcal{M}$ equipped with the usual supremum norm. By the Stone-Weierstrass Theorem, the set of finite sums of functions of the form $\bar{\varphi} \psi$, with $\varphi, \psi \in H^{\infty}$, is dense in $C(\mathcal{M})$. Identifying a point in the unit disk $\mathbb{D}$ with the functional of evaluation at this point, we regard $\mathbb{D}$ as a subset of $\mathcal{M}$. Carleson's Corona Theorem states that $\mathbb{D}$ is a dense subset of $\mathcal{M}$. The pseudohyperbolic distance between $m$ and $m^{\prime}$ in $\mathcal{M}$ is defined by

$$
d\left(m, m^{\prime}\right)=\sup \left\{\left|m^{\prime}(h)\right|: h \in H^{\infty},\|h\|_{\infty} \leq 1, \text { and } m(h)=0\right\} .
$$

For $z, w \in \mathbb{D}$ we have $d(z, w)=\left|\varphi_{w}(z)\right|$. For $m$ in $\mathcal{M}$ the Gleason part containing $m$ in $\mathcal{M}$ is the set $G(m)=\left\{m^{\prime} \in \mathcal{M}: d\left(m, m^{\prime}\right)<1\right\}$. The Gleason parts form a partition of $\mathcal{M}$, and for each $w \in \mathbb{D}$ the Gleason part containing $w$ is equal to $\mathbb{D}$. For each $m \in \mathcal{M}$, Hoffman [13] constructed a canonical continuous map $\varphi_{m}: \mathbb{D} \rightarrow \mathcal{M}$ such that $\varphi_{m}(0)=m$. The map $\varphi_{m}$ is defined by setting

$$
\varphi_{m}(w) h=\lim _{z \rightarrow m} h \circ \varphi_{z}(w),
$$

for $w \in \mathbb{D}$ and $h \in H^{\infty}$, where the limit is taken in $\mathcal{M}$. Hoffman [13] proved the existence of this limit, as well as many other deep properties of the mappings $\varphi_{m}$. For an exposition of Hoffman's result see [10], Chapter X. We recall here the following properties. The image of $\varphi_{m}$ is the Gleason part $G(m)$, and $\varphi_{m}$ is injective if $G(m)$ consists of more than one point. If $f \in C(\mathcal{M}), m \in \mathcal{M}$ and $\left(w_{j}\right)$ is a net in $\mathbb{D}$ converging to $m$ in $\mathcal{M}$, then $f \circ \varphi_{w_{j}} \rightarrow f \circ \varphi_{m}$ uniformly on each compact subset of $\mathbb{D}$ (see, for example, [19], Lemma 5). In particular, if $f \in H^{\infty}$ and $m \in \mathcal{M}$, then the composition $f \circ \varphi_{m}$ is in $H^{\infty}$. 
If $G$ is a Gleason part and $f$ is a function on $\mathcal{M}$, we say that $f$ is analytic on $G$ if the function $f \circ \varphi_{m}$ is analytic on $\mathbb{D}$, where $m \in G$. Note that this definition is independent of the chosen representative $m$ for the Gleason part $G$ : if $m^{\prime} \in G$ is distinct from $m$, then $m^{\prime}=\varphi_{m}(\lambda)$, for some $\lambda \in \mathbb{D}$. By the Schwarz Lemma there is a unimodular constant $\zeta$ for which $\varphi_{m^{\prime}}(z)=\varphi_{m}\left(\varphi_{\lambda}(\zeta z)\right)$, for all $z \in \mathbb{D}$. It follows that $f \circ \varphi_{m^{\prime}}$ is analytic on $\mathbb{D}$ if and only if $f \circ \varphi_{m}$ is analytic on $\mathbb{D}$.

We have the following characterization of essentially commuting dual Toeplitz operators. Note that this result is analogous to the characterization of essentially commuting Toeplitz operators on the Bergman space obtained by the first author [19] as well as the characterization of essentially commuting Toeplitz operators on the Hardy space obtained by Gorkin and the second author [11.

Theorem 5.1. Let $f$ and $g$ be in $C(\mathcal{M})$. Then: $S_{f}$ and $S_{g}$ are essentially commuting if and only if on each of the Gleason parts $G$ in $\mathcal{M} \backslash \mathbb{D}$ one of the following conditions holds:

(i) both $f$ and $g$ are analytic on $G$;

(ii) both $\bar{f}$ and $\bar{g}$ are analytic on $G$;

(iii) there are constants $\alpha$ and $\beta$, not both zero, such that the function $\alpha f+\beta g$ is constant on $G$.

Proof. Suppose that $m \in \mathcal{M} \backslash \mathbb{D}$. Let $\left(w_{j}\right)$ be a net in $\mathbb{D}$ converging to $m$. First assume that there exists a $\delta>0$ such that $\left\|H_{f} k_{w_{j}}\right\|_{2} \geq \delta$ and $\left\|H_{\bar{f}} k_{w_{j}}\right\|_{2} \geq \delta$ for all indices $j$. The numbers $\lambda_{j}=\left\langle H_{g} k_{w_{j}}, H_{f} k_{w_{j}}\right\rangle /\left\|H_{f} k_{w_{j}}\right\|_{2}^{2}$ are bounded by $M=\|f\|_{\infty}\|g\|_{\infty} / \delta^{2}$. Passing to a subnet, if necessary, we may assume that $\lambda_{j} \rightarrow \lambda$. It is easily seen that then $\left(g-\lambda_{j} f\right) \circ \varphi_{w_{j}} \rightarrow(g-\lambda f) \circ \varphi_{m}$ pointwise on $\mathbb{D}$. We claim that this convergence is also in $L^{2}(\mathbb{D}, d A)$. Some care needs to be taken to prove this claim, since the bounded convergence theorem does not hold for nets. A standard density argument shows that the convergence $\left(g-\lambda_{j} f\right) \circ \varphi_{w_{j}} \rightarrow(g-\lambda f) \circ \varphi_{m}$ is uniform on compact subsets of $\mathbb{D}$ (see [19, Lemma 5). Using that

$$
\begin{aligned}
& \left\|\left(g-\lambda_{j} f\right) \circ \varphi_{w_{j}}-(g-\lambda f) \circ \varphi_{m}\right\|_{2}^{2} \\
& \quad \leq \sup _{|z| \leq r}\left|\left(g-\lambda_{j} f\right) \circ \varphi_{w_{j}}(z)-(g-\lambda f) \circ \varphi_{m}(z)\right|^{2}+4\left(1-r^{2}\right)\left(\|f\|_{\infty}+M\|g\|_{\infty}\right)^{2},
\end{aligned}
$$

for all $0<r<1$, we see that indeed $\left(g-\lambda_{j} f\right) \circ \varphi_{w_{j}} \rightarrow(g-\lambda f) \circ \varphi_{m}$ in $L^{2}(\mathbb{D}, d A)$. Thus $\left\|Q\left(\left(g-\lambda_{j} f\right) \circ \varphi_{w_{j}}\right)\right\|_{2} \rightarrow\left\|Q\left((g-\lambda f) \circ \varphi_{m}\right)\right\|_{2}$. Since $\left\|H_{g-\lambda_{j} f} k_{w_{j}}\right\|_{2}=$ $\left\|Q\left(\left(g-\lambda_{j} f\right) \circ \varphi_{w_{j}}\right)\right\|_{2}$ (see [21]) we obtain

$$
\left\|H_{g-\lambda_{j} f} k_{w_{j}}\right\|_{2} \rightarrow\left\|Q\left((g-\lambda f) \circ \varphi_{m}\right)\right\|_{2} .
$$

Likewise

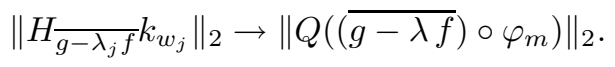

Since also $\left\|H_{f} k_{w_{j}}\right\|_{2} \rightarrow\left\|Q\left(f \circ \varphi_{m}\right)\right\|_{2}$ and $\left\|H_{\bar{f}} k_{w_{j}}\right\|_{2} \rightarrow\left\|Q\left(\bar{f} \circ \varphi_{m}\right)\right\|_{2}$, it follows that

$$
\begin{aligned}
& \left\|H_{f} k_{w_{j}}\right\|_{2}\left\|H_{\overline{g-\lambda_{j} f}} k_{w_{j}}\right\|_{2}+\left\|H_{\bar{f}} k_{w_{j}}\right\|_{2}\left\|H_{g-\lambda_{j} f} k_{w_{j}}\right\|_{2} \\
& \quad \rightarrow\left\|Q\left(f \circ \varphi_{m}\right)\right\|_{2}\left\|Q\left((\overline{g-\lambda f}) \circ \varphi_{m}\right)\right\|_{2}+\left\|Q\left(\bar{f} \circ \varphi_{m}\right)\right\|_{2}\left\|Q\left((g-\lambda f) \circ \varphi_{m}\right)\right\|_{2} .
\end{aligned}
$$

If $\left\|F_{w_{j}}\right\| \rightarrow 0$, then appealing to Lemma 4.4 we get

$$
\left\|Q\left(f \circ \varphi_{m}\right)\right\|_{2}\left\|Q\left((\overline{g-\lambda f}) \circ \varphi_{m}\right)\right\|_{2}+\left\|Q\left(\bar{f} \circ \varphi_{m}\right)\right\|_{2}\left\|Q\left((g-\lambda f) \circ \varphi_{m}\right)\right\|_{2}=0 .
$$


Since $\left\|Q\left(f \circ \varphi_{m}\right)\right\|_{2} \geq \delta>0$ and $\left\|Q\left(\bar{f} \circ \varphi_{m}\right)\right\|_{2} \geq \delta>0$, we conclude that $\left\|Q\left((\overline{g-\lambda f}) \circ \varphi_{m}\right)\right\|_{2}=\left\|Q\left((g-\lambda f) \circ \varphi_{m}\right)\right\|_{2}=0$. This implies that the function $(g-\lambda f) \circ \varphi_{m}$ is both analytic and co-analytic, thus is constant. Conversely, if $(g-\lambda f) \circ \varphi_{m}$ is constant, then the above argument shows that necessarily $\left\|F_{w_{j}}\right\| \rightarrow 0$.

In case the numbers $\left\|H_{f} k_{w_{j}}\right\|_{2}$ are not bounded below, by passing to a subnet we may assume that $\left\|H_{f} k_{w_{j}}\right\|_{2} \rightarrow 0$, and thus $\left\|Q\left(f \circ \varphi_{m}\right)\right\|_{2}=0$. This means that $f \circ \varphi_{m}$ is analytic on $\mathbb{D}$. Since

$$
\left\|F_{w_{j}}\right\|-\left\|H_{g} k_{w_{j}}\right\|_{2}\left\|H_{\bar{f}} k_{w_{j}}\right\|_{2} \rightarrow 0
$$

we have

$$
\left\|F_{w_{j}}\right\| \rightarrow\left\|Q\left(g \circ \varphi_{m}\right)\right\|_{2}\left\|Q\left(\bar{f} \circ \varphi_{m}\right)\right\|_{2} .
$$

From this we see: $\left\|F_{w_{j}}\right\| \rightarrow 0$ if and only if $\left\|Q\left(g \circ \varphi_{m}\right)\right\|_{2}=0$ or $\left\|Q\left(\bar{f} \circ \varphi_{m}\right)\right\|_{2}=0$. In case $\left\|Q\left(g \circ \varphi_{m}\right)\right\|_{2}=0$ the function $g \circ \varphi_{m}$ is analytic, so that case (i) occurs; in case $\left\|Q\left(\bar{f} \circ \varphi_{m}\right)\right\|_{2}=0$ the function $\bar{f} \circ \varphi_{m}$ is analytic, so that $f \circ \varphi_{m}$ must be constant and case (iii) occurs.

The case where the numbers $\left\|H_{\bar{f}} k_{w_{j}}\right\|_{2}$ are not bounded below is handled similarly.

An operator $S$ is essentially normal if the commutator $\left[S, S^{*}\right]=S S^{*}-S^{*} S$ is compact. The above theorem has the following consequence.

Corollary 5.2. Let $f$ be in $C(\mathcal{M})$. Then: $S_{f}$ is essentially normal if and only if $f$ maps each Gleason part of $\mathcal{M}$, except $\mathbb{D}$, into a line in the complex plane.

Proof. Observe that if $G$ is a Gleason part of $\mathcal{M}$ not equal to $\mathbb{D}$, then $f(G)$ is contained in a line in the complex plane if and only if there exist constants $\alpha$ and $\beta$, not both 0 , such that $\alpha f+\beta \bar{f}$ is constant on $G$.

\section{The Commutator Ideal}

Writing $\mathcal{B}\left(L_{a}^{2}\right)$ for the set of all bounded operators on $L_{a}^{2}$, the Toeplitz algebra $\mathcal{T}$ is the $C^{*}$-subalgebra of $\mathcal{B}\left(L_{a}^{2}\right)$ generated by all Toeplitz operators $T_{g}$ for $g \in H^{\infty}$. If we let $\mathcal{U}$ denote the $C^{*}$-subalgebra of $L^{\infty}(\mathbb{D}, d A)$ generated by $H^{\infty}$, then $\mathcal{U}$ equals the closed subalgebra of $L^{\infty}(\mathbb{D}, d A)$ generated by the set of bounded harmonic functions on $\mathbb{D}$ (see [2, Proposition 4.5), and it can be shown that $\mathcal{T}$ is equal to the closed subalgebra of $\mathcal{B}\left(L_{a}^{2}\right)$ generated by all Toeplitz operators $T_{u}$ with $u \in \mathcal{U}$.

Recall that the commutator ideal $\mathcal{C}_{\mathcal{T}}$ is the smallest closed, two-sided ideal of $\mathcal{T}$ containing all commutators $[R, S]=R S-S R$, where $R, S \in \mathcal{T}$. McDonald and Sundberg [15] showed that $\mathcal{T} / \mathcal{C}_{\mathcal{T}}$ is isomorphic, as a $C^{*}$-algebra, to $C\left(\mathcal{M}_{1}\right)$, where $\mathcal{M}_{1}$ denotes the subset of $\mathcal{M}$ consisting of all one-point parts (that is, the set of $m \in$ $\mathcal{M}$ for which $G(m)=\{m\})$. More precisely, they proved that the map $u \mapsto T_{u}+\mathcal{C}_{\mathcal{T}}$ is a surjective homomorphism of $\mathcal{U}$ onto $\mathcal{T} / \mathcal{C}_{\mathcal{T}}$, with kernel $\left\{u \in \mathcal{U}:\left.u\right|_{\mathcal{M}_{1}}=0\right\}$. This theorem says that each $S \in \mathcal{T}$ can be written in the form $S=T_{u}+R$ for some $u \in \mathcal{U}$ and $R \in \mathcal{C}_{\mathcal{T}}$, and that for every $u \in \mathcal{U}$ the Toeplitz operator $T_{u}$ belongs to the commutator ideal $\mathcal{C}_{\mathcal{T}}$ if and only if $\left.u\right|_{\mathcal{M}_{1}}=0$. These results account for the importance of understanding the commutator ideal $\mathcal{C}_{\mathcal{T}}$. The decomposition of $S \in \mathcal{T}$ as $S=T_{u}+R$ with $u \in \mathcal{U}$ and $R \in \mathcal{C}_{\mathcal{T}}$ is not unique. The Berezin transform of an operator $S \in \mathcal{B}\left(L_{a}^{2}\right)$ is defined by $\tilde{S}(z)=\left\langle S k_{z}, k_{z}\right\rangle$, for $z \in \mathbb{D}$. Axler and the second author [4] used the Berezin transform to obtain a canonical decomposition 
of a given operator in $\mathcal{T}$, and showed that $S-T_{\tilde{S}}$ is in the commutator ideal $\mathcal{C}_{\mathcal{T}}$ for every $S \in \mathcal{T}$. Furthermore, an operator $S \in \mathcal{B}\left(L_{a}^{2}\right)$ is in the commutator ideal $\mathcal{C}_{\mathcal{T}}$ if and only if $\left.\tilde{S}\right|_{\mathcal{M}_{1}}=0$ (here $\tilde{S}$ is the continuous extension to the maximal ideal space of the Berezin transform of $S$; see 4]). Writing $S=T_{\tilde{S}}+\left(S-T_{\tilde{S}}\right)$ gives a canonical way to express the (nondirect) sum $\mathcal{T}=\left\{T_{u}: u \in \mathcal{U}\right\}+\mathcal{C}_{\mathcal{T}}$.

Denoting the set of bounded operators on $\left(L_{a}^{2}\right)^{\perp}$ by $\mathcal{B}\left(\left(L_{a}^{2}\right)^{\perp}\right)$, the dual Toeplitz algebra $\mathcal{D} \mathcal{T}$ is the $C^{*}$-subalgebra of $\mathcal{B}\left(\left(L_{a}^{2}\right)^{\perp}\right)$ generated by the dual Toeplitz operators $S_{f}$ for $f \in H^{\infty}$. Let $\mathcal{C}_{\mathcal{D} \mathcal{T}}$ be the commutator ideal of $\mathcal{D} \mathcal{T}$. We have the following analogue of the McDonald-Sundberg theorem:

Theorem 6.1. The map $\Psi: C(\mathcal{M}) \rightarrow \mathcal{D} \mathcal{T} / \mathcal{C}_{\mathcal{D T}}$ defined by

$$
\Psi(f)=S_{f}+\mathcal{C}_{\mathcal{D} \mathcal{T}}
$$

is a $C^{*}$-algebra isomorphism.

This theorem states that each $S \in \mathcal{D} \mathcal{T}$ can be written in the form $S=S_{f}+R$, where $f \in C(\mathcal{M})$ and $R \in \mathcal{C}_{\mathcal{D T}}$. Unlike the McDonald-Sundberg theorem for the Toeplitz algebra, this decomposition is unique. Consequently, the only dual Toeplitz operator contained in the commutator ideal $\mathcal{C}_{\mathcal{D} \mathcal{T}}$ is the zero operator.

Proof. Using the elementary algebraic properties of dual Toeplitz operators from the beginning of Section 2, it is obvious that $\Psi$ is a linear ${ }^{*}$-map into $\mathcal{D T} / \mathcal{C}_{\mathcal{D T}}$. Next we will show that the mapping $\Psi$ is multiplicative. Since sums of elements of the form $f \bar{g}$, for $f$ and $g$ in $H^{\infty}$, are dense in $C(\mathcal{M})$, it is sufficient to show that

$$
\Psi(f \bar{g} h \bar{k})=\Psi(f \bar{g}) \Psi(h \bar{k}),
$$

for $f, g, h$ and $k$ in $H^{\infty}$. This is equivalent to showing that

$$
S_{f \bar{g}} S_{h \bar{k}}-S_{f \bar{g} h \bar{k}} \in \mathcal{C}_{\mathcal{D} \mathcal{T}} .
$$

Using (2.2) we have

$$
S_{f \bar{g}} S_{h \bar{k}}-S_{f \bar{g} h \bar{k}}=S_{f}\left(S_{\bar{g}} S_{h}-S_{h} S_{\bar{g}}\right) S_{\bar{k}}
$$

so that indeed $S_{f \bar{g}} S_{h \bar{k}}-S_{f \bar{g} h \bar{k}} \in \mathcal{C}_{\mathcal{D T}}$. This completes the proof that $\Psi$ is multiplicative.

Thus $\mathcal{D} \mathcal{T} / \mathcal{C}_{\mathcal{D} \mathcal{T}}$ is ${ }^{*}$-isometrically isomorphic to $C(\mathcal{M}) / \operatorname{ker} \Psi$, where

$$
\operatorname{ker} \Psi=\left\{\varphi \in C(\mathcal{M}): \varphi=0 \text { on } \mathcal{M}^{\prime}\right\}
$$

for some closed subset $\mathcal{M}^{\prime}$ of $\mathcal{M}$.

To finish the proof, we need to identify the subset $\mathcal{M}^{\prime}$. We shall show that $\mathcal{M}^{\prime}=\mathcal{M}$.

First we show that $\mathcal{M}^{\prime}$ contains the unit disk $\mathbb{D}$. Since $\mathcal{D} \mathcal{T}$ is generated by subnormal operators, a theorem of Bunce [7] shows that for all $f \in H^{\infty}$ the left spectrum of $S_{\bar{f}}$ is identified with the set $\overline{f\left(\mathcal{M}^{\prime}\right)}$. So it suffices to show that for each $w$ in $\mathbb{D}$ and $f \in H^{\infty}, \overline{f(w)}$ is in the left spectrum of $S_{\bar{f}}$. To do this, for $w \in \mathbb{D}$ and $0<s<1-|w|$, let $g_{w, s}$ be the function on $\mathbb{D}$ defined by $g_{w, s}(z)=(\overline{z-w}) \chi_{w+s \mathbb{D}}(z)$, for $z \in \mathbb{D}$. For each $h \in L_{a}^{2}$,

$$
\int_{\mathbb{D}} h(z) \overline{g_{w, s}(z)} d A(z)=\int_{s \mathbb{D}} h(z+w) z d A(z)=0 .
$$

Thus $g_{w, s} \in\left(L_{a}^{2}\right)^{\perp}$ and $(\overline{z-w}) g_{w, s} \in\left(L_{a}^{2}\right)^{\perp}$. An easy computation yields

$$
\left\|S_{\overline{z-w}} g_{w, s}\right\|_{2}=\left\|(\overline{z-w}) g_{s}\right\|_{2}=s^{3} / \sqrt{3}
$$


On the other hand, $\left\|g_{w, s}\right\|_{2}=s^{2} / \sqrt{2}$. Therefore

$$
\left\|S_{\overline{z-w}}\left(g_{w, s} /\left\|g_{w, s}\right\|_{2}\right)\right\|_{2}=s \sqrt{2 / 3} .
$$

For each $f \in H^{\infty}$, there is a function $f_{1} \in H^{\infty}$ such that $f(z)-f(w)=(z-w) f_{1}(z)$, for all $z \in \mathbb{D}$. Then

$$
\begin{aligned}
\left\|S_{\overline{f-f(w)}}\left(g_{s} /\left\|g_{s}\right\|_{2}\right)\right\|_{2} & =\left\|S_{\bar{f}_{1}} S_{\overline{z-w}}\left(g_{s} /\left\|g_{s}\right\|_{2}\right)\right\|_{2} \\
& \leq\left\|f_{1}\right\|_{\infty}\left\|S_{\overline{z-w}}\left(g_{s} /\left\|g_{s}\right\|_{2}\right)\right\|_{2} \\
& =\left\|f_{1}\right\|_{\infty} s \sqrt{2 / 3} \rightarrow 0,
\end{aligned}
$$

as $s \rightarrow 0^{+}$. This implies that $\overline{f(w)}$ is in the left spectrum of $S_{\bar{f}}$.

By Carleson's Corona Theorem the unit disk $\mathbb{D}$ is dense in $\mathcal{M}$. Thus $\mathcal{M}^{\prime}=\mathcal{M}$, so that $\Psi$ is injective. The range of $\Psi$ is easily seen to be dense in $\mathcal{D T} / \mathcal{C}_{\mathcal{D} \mathcal{T}}$. Thus $\Psi$ is also surjective.

McDonald and Sundberg 15 proved that a Toeplitz operator with symbol in $C(\mathcal{M})$ is only compact if the symbol is identically equal to 0 . We have the following analogous result for dual Toeplitz operators.

Corollary 6.2. If $f \in C(\mathcal{M})$, then $S_{f}$ is compact if and only if $f=0$ on $\mathcal{M}$.

Proof. Let $f \in C(\mathcal{M})$ and suppose that the operator $S_{f}$ is compact. For $w \in \mathbb{D}$ and $0<s<1-|w|$ let $g_{w, s}$ be the function defined in the proof of Theorem 6.1 and let $u_{w, s}=g_{w, s} /\left\|g_{w, s}\right\|_{2}$. The functions $u_{w, s}$ are unit vectors in the space $\left(L_{a}^{2}\right)^{\perp}$. For $\psi \in L^{2}(\mathbb{D}, d A)$, applying the Cauchy-Schwarz inequality, we have

$$
\left|\left\langle\psi, g_{w, s}\right\rangle\right|=\left|\int_{w+s \mathbb{D}} \psi(z) \overline{g_{w, s}(z)} d A(z)\right| \leq\left\|g_{w, s}\right\|_{2}\left(\int_{w+s \mathbb{D}}|\psi(z)|^{2} d A(z)\right)^{1 / 2} .
$$

Thus

$$
\left|\left\langle\psi, u_{w, s}\right\rangle\right| \leq\left(\int_{w+s \mathbb{D}}|\psi(z)|^{2} d A(z)\right)^{1 / 2}
$$

and it follows that the functions $u_{w, s}$ tend to 0 weakly in $L^{2}(\mathbb{D}, d A)$, and thus in $\left(L_{a}^{2}\right)^{\perp}$, as $s \rightarrow 0^{+}$. Since $S_{f}$ is compact, we must have $\left\|S_{f} u_{w, s}\right\|_{2} \rightarrow 0$ as $s \rightarrow 0^{+}$. Using the continuity of $f$ at $w$ it is easily seen that $\left\|S_{f-f(w)} u_{w, s}\right\|_{2} \rightarrow 0$, thus

$$
\left\|S_{f} u_{w, s}\right\|_{2} \rightarrow|f(w)|
$$

as $s \rightarrow 0^{+}$. Hence $f(w)=0$. Since $\mathbb{D}$ is dense in $\mathcal{M}$ and $f$ is continuous, we conclude that $f=0$.

In the next section we will generalize the above result to arbitrary bounded measurable symbols.

\section{Bounded and Compact Dual Toeplitz Operators}

For $f \in L^{2}(\mathbb{D}, d A)$ the dual Toeplitz operator $S_{f}$ is densely defined by the formula $S_{f} u=Q(f u)$, for $u \in\left(L_{a}^{2}\right)^{\perp} \cap L^{\infty}(\mathbb{D}, d A)$. In this section we will characterize the bounded and compact dual Toeplitz operators.

The functions $u_{w, s}$ in the following lemma are defined in the proof of Corollary 6.2 
Lemma 7.1. Let $f \in L^{2}(\mathbb{D}, d A)$. For each $w \in \mathbb{D}$,

$$
\lim _{s \rightarrow 0^{+}}\left\|H_{f}^{*} u_{w, s}\right\|_{2}=0 .
$$

Proof. Fix $w \in \mathbb{D}$. For every $z \in \mathbb{D}$ we have

$$
\begin{aligned}
H_{f}^{*} g_{w, s}(z) & =\int_{\mathbb{D}} \overline{f(\lambda)} g_{w, s}(\lambda) K_{z}(\lambda) d A(\lambda) \\
& =\int_{w+s \mathbb{D}} \overline{f(\lambda)} g_{w, s}(\lambda) K_{z}(\lambda) d A(\lambda) .
\end{aligned}
$$

The Cauchy-Schwarz inequality gives

$$
\left|H_{f}^{*} g_{w, s}(z)\right| \leq\left\|g_{s, w}\right\|_{2}\left(\int_{w+s \mathbb{D}}|f(\lambda)|^{2}\left|K_{z}(\lambda)\right|^{2} d A(\lambda)\right)^{1 / 2}
$$

so that

$$
\left|H_{f}^{*} u_{w, s}(z)\right|^{2} \leq \int_{w+s \mathbb{D}}|f(\lambda)|^{2}\left|K_{z}(\lambda)\right|^{2} d A(\lambda),
$$

for all $z \in \mathbb{D}$. Integrate with respect to $z$ to obtain

$$
\left\|H_{f}^{*} u_{w, s}\right\|_{2}^{2} \leq \int_{w+s \mathbb{D}}|f(\lambda)|^{2}\left\{\int_{\mathbb{D}}\left|K_{z}(\lambda)\right|^{2} d A(z)\right\} d A(\lambda)
$$

and using $\int_{\mathbb{D}}\left|K_{z}(\lambda)\right|^{2} d A(z)=\int_{\mathbb{D}}\left|K_{\lambda}(z)\right|^{2} d A(z)=K_{\lambda}(\lambda)=1 /\left(1-|\lambda|^{2}\right)^{2}$, we get

$$
\begin{aligned}
\left\|H_{f}^{*} u_{w, s}\right\|_{2}^{2} & \leq \int_{w+s \mathbb{D}} \frac{|f(\lambda)|^{2}}{\left(1-|\lambda|^{2}\right)^{2}} d A(\lambda) \\
& \leq \frac{1}{(1-(|w|+s))^{2}} \int_{w+s \mathbb{D}}|f(\lambda)|^{2} d A(\lambda),
\end{aligned}
$$

which gives the stated result, since $\int_{w+s \mathbb{D}}|f(\lambda)|^{2} d A(\lambda) \rightarrow 0$ as $s \rightarrow 0^{+}$.

The following lemma will be used repeatedly.

Lemma 7.2. For $f \in L^{2}(\mathbb{D}, d A)$ we have

$$
|f(w)|=\lim _{s \rightarrow 0^{+}}\left\|S_{f} u_{w, s}\right\|_{2},
$$

for almost every $w \in \mathbb{D}$.

Proof. Note that $M_{f} u=S_{f} u+H_{\bar{f}}^{*} u$, and $S_{f} u \perp H_{\bar{f}}^{*} u$ for every bounded $u \in\left(L_{a}^{2}\right)^{\perp}$. Thus

$$
\left\|M_{f} u\right\|_{2}^{2}=\left\|S_{f} u\right\|_{2}^{2}+\left\|H_{\bar{f}}^{*} u\right\|_{2}^{2} .
$$

For each $w \in \mathbb{D}$, taking $u=u_{w, s}$ in the above equality, by Lemma 7.1 we have

$$
\lim _{s \rightarrow 0^{+}}\left\|S_{f} u_{w, s}\right\|_{2}^{2}=\lim _{s \rightarrow 0^{+}}\left\|M_{f} u_{w, s}\right\|_{2}^{2} .
$$

We claim that

$$
\lim _{s \rightarrow 0^{+}}\left\|M_{f} u_{w, s}\right\|_{2}^{2}=\lim _{s \rightarrow 0^{+}} \frac{\int_{|z-w|<s}|f(z)|^{2}|z-w|^{2} d A(z)}{\int_{|z-w|<s}|z-w|^{2} d A(z)}=|f(w)|^{2},
$$


for a.e. $w \in \mathbb{D}$. Clearly this claim will prove the stated result. To prove (7.3) write $g=|f|^{2}$. Using the fact that $\int_{|z-w|<s}|z-w|^{2} d A(z)=s^{4} / 2$, we have

$$
\begin{aligned}
\left|\frac{\int_{|z-w|<s} g(z)|z-w|^{2} d A(z)}{\int_{|z-w|<s}|z-w|^{2} d A(z)}-g(w)\right| & \leq \frac{s^{2} \int_{|z-w|<s}|g(z)-g(w)| d A(z)}{s^{4} / 2} \\
& =\frac{2}{|B(w, s)|} \int_{B(w, s)}|g(z)-g(w)| d A(z),
\end{aligned}
$$

where $B(w, s)=\{z \in \mathbb{C}:|z-w|<s\}$, so (7.3) holds for all $w$ in the Lebesgue set

$$
\left\{w \in \mathbb{D}: \lim _{s \rightarrow 0^{+}} \frac{1}{|B(w, s)|} \int_{B(w, s)}|g(z)-g(w)| d A(z)=0\right\} .
$$

It is a classical theorem of Lebesgue that the complement of the above set in $\mathbb{D}$ has area measure 0 (see, for example, Theorem 8.8 in [17]).

For $f \in L^{2}(\mathbb{D}, d A)$ we can consider $T_{f}$ and $S_{f}$ as densely defined operators on $L_{a}^{2}$ and $\left(L_{a}^{2}\right)^{\perp}$, respectively. It is well-known that there exist unbounded functions $f$ for which the Toeplitz operator $T_{f}$ is bounded on $L_{a}^{2}$. In contrast, for dual Toeplitz operators we have the following result.

Theorem 7.4. Let $f \in L^{2}(\mathbb{D}, d A)$. Then $S_{f}$ is bounded if and only if $f \in L^{\infty}(\mathbb{D})$, in which case $\left\|S_{f}\right\|=\|f\|_{\infty}$.

Proof. The "if" part is trivial: if $f \in L^{\infty}(\mathbb{D}, d A)$, then $S_{f}$ is bounded with $\left\|S_{f}\right\| \leq$ $\|f\|_{\infty}$. To prove the "only if" part, suppose that $S_{f}$ is bounded. Then $\left\|S_{f} u_{w, s}\right\| \leq$ $\left\|S_{f}\right\|$, for all $w \in \mathbb{D}$ and $0<s<1-|w|$. It follows from Lemma 7.2 that $\|f\|_{\infty} \leq$ $\left\|S_{f}\right\|$.

Brown and Halmos showed that the only compact (completely continuous) Toeplitz operator on the Hardy space is the zero operator. This is easily seen to be false for Toeplitz operators on the Bergman space: for every continuous function $f$ on $\mathbb{D}$ such that $f(w) \rightarrow 0$ as $|w| \rightarrow 1^{-}$the Toeplitz operator $T_{f}$ is compact on $L_{a}^{2}$. A complete characterization of compact Toeplitz operators on the Bergman space via the Berezin transform has recently been obtained by Axler and the second author [5. In contrast with Toeplitz operators on the Bergman space we have the following result for dual Toeplitz operators.

Theorem 7.5. For $f$ in $L^{\infty}(\mathbb{D}, d A): S_{f}$ is compact if and only if $f=0$ a.e. on $\mathbb{D}$.

Proof. Since $u_{w, s} \rightarrow 0$ weakly in $\left(L_{a}^{2}\right)^{\perp}$, if $S_{f}$ is compact, then for each $w \in \mathbb{D}$ we have $\left\|S_{f} u_{w, s}\right\|_{2} \rightarrow 0$ as $s \rightarrow 0^{+}$, and it follows from Lemma 7.2 that $f(w)=0$ for a.e. $w \in \mathbb{D}$.

We conclude this section with the following generalization of Theorem 3.1.

Theorem 7.6. Let $f$ and $g$ be in $L^{\infty}(\mathbb{D})$. If $S_{f} S_{g}$ is a compact perturbation of a dual Toeplitz operator $S_{h}$, then $f(w) g(w)=h(w)$ for almost all $w \in \mathbb{D}$, and $H_{f} H_{\bar{g}}^{*}$ is compact.

Proof. Assume that $S_{f} S_{g}-S_{h}$ is compact. Then, using (2.1), we see that the operator

$$
S_{f g-h}-H_{f} H_{\bar{g}}^{*}=S_{f} S_{g}-S_{h}
$$


is compact. For $w \in \mathbb{D}, u_{w, s} \rightarrow 0$ weakly in $\left(L_{a}^{2}\right)^{\perp}$ as $s \rightarrow 0^{+}$; thus

$$
\left\|\left(S_{f g-h}-H_{f} H_{\bar{g}}^{*}\right) u_{w, s}\right\|_{2} \rightarrow 0 .
$$

By Lemma 7.1 we also have

$$
\left\|H_{f} H_{\bar{g}}^{*} u_{w, s}\right\|_{2} \rightarrow 0
$$

Thus

$$
\left\|S_{f g-h} u_{w, s}\right\|_{2} \rightarrow 0
$$

Applying Lemma 7.2 we see that

$$
\left\|S_{f g-h} u_{w, s}\right\|_{2}^{2} \rightarrow|f(w) g(w)-h(w)|^{2},
$$

for a.e. $w$ in $\mathbb{D}$. Since $f(w) g(w)-h(w)=0$ for almost all $w \in \mathbb{D}$, we have that $S_{f g-h}=0$. Hence $H_{f} H_{\bar{g}}^{*}$ is compact.

\section{Symbol Map on the Dual Toeplitz Algebra}

The symbol map on the Toeplitz algebra in the Hardy space setting is described in Chapter 7 of $[9$. In this section we will show the existence of a symbol map on the dual Toeplitz algebra. Our construction of this symbol map will make use of the following lemma.

Lemma 8.1. If the operator $S$ is in the closed ideal generated by the semicommutators of all bounded dual Toeplitz operators, then

$$
\left\|S u_{w, s}\right\|_{2} \rightarrow 0
$$

for all $w$ in $\mathbb{D}$ as $s \rightarrow 0^{+}$.

Proof. If operator $S$ is in the closed ideal generated by the semicommutators of all bounded dual Toeplitz operators, then $S$ can be approximated by a finite sum of finite products of dual Toeplitz operators or operators of the form $S_{f g}-S_{f} S_{g}$. Noting that

$$
S_{f g}-S_{f} S_{g}=H_{f} H_{\bar{g}}^{*}
$$

Lemma 7.1 gives that

$$
\left\|\left(S_{f g}-S_{f} S_{g}\right) u_{w, s}\right\|_{2} \rightarrow 0,
$$

for all $w$ in $\mathbb{D}$ as $s \rightarrow 0^{+}$. To prove the stated result, it suffices to show that for $f, g$, and $h_{1}, \ldots, h_{n}$ in $L^{\infty}(\mathbb{D})$,

$$
\left\|\left(S_{f g}-S_{f} S_{g}\right) S_{h_{1}} S_{h_{2}} \cdots S_{h_{n-1}} S_{h_{n}} u_{w, s}\right\|_{2} \rightarrow 0,
$$

for all $w$ in $\mathbb{D}$ as $s \rightarrow 0^{+}$. This can be proved using induction. To prove the basis for the induction put $h=h_{1}$. Repeatedly using (2.1) we gave

$$
\begin{aligned}
\left(S_{f g}-S_{f} S_{g}\right) S_{h} & =S_{f g} S_{h}-S_{f} S_{g} S_{h}=S_{f g} S_{h}-S_{f}\left(S_{g h}-H_{g} H_{\bar{h}}^{*}\right) \\
& =S_{f g} S_{h}-S_{f} S_{g h}+S_{f} H_{g} H_{\bar{h}}^{*} \\
& =\left(S_{f g h}-S_{f} S_{g h}\right)-\left(S_{f g h}-S_{f g} S_{h}\right)+S_{f} H_{g} H_{\bar{h}}^{*} \\
& =H_{f} H_{g h}^{*}-H_{f g} H_{\bar{h}}^{*}+S_{f} H_{g} H_{\bar{h}}^{*} .
\end{aligned}
$$


Using Lemma 7.1 we conclude that $\left\|\left(S_{f g}-S_{f} S_{g}\right) S_{h} u_{w, s}\right\|_{2} \rightarrow 0$, for all $w$ in $\mathbb{D}$ as $s \rightarrow 0^{+}$. The case $n=$ can be proved similarly. The induction step follows likewise from the observation that

$$
\begin{aligned}
\left(S_{f g}-S_{f} S_{g}\right) S_{h_{1}} S_{h_{2}} \cdots S_{h_{n-1}} S_{h_{n}}= & \left(S_{f g}-S_{f} S_{g}\right) S_{h_{1}} S_{h_{2}} \cdots S_{h_{n-1} h_{n}} \\
& -\left(S_{f g}-S_{f} S_{g}\right) S_{h_{1}} S_{h_{2}} \cdots S_{h_{n-2}} H_{h_{n-1}} H_{h_{n}}^{*},
\end{aligned}
$$

for $n>2$.

Proposition 8.2. For $f_{1}, f_{2}, \ldots, f_{n} \in L^{\infty}(\mathbb{D})$ the operator

$$
S_{f_{1}} S_{f_{2}} \cdots S_{f_{n}}-S_{f_{1} f_{2} \cdots f_{n}}
$$

belongs to the closed ideal generated by the semicommutators of all bounded dual Toeplitz operators.

Proof. Writing

$$
S_{f_{1}} S_{f_{2}} \cdots S_{f_{n}}-S_{f_{1} f_{2} \cdots f_{n}}=S_{f_{1}}\left(S_{f_{2}} \cdots S_{f_{n}}-S_{f_{2} \cdots f_{n}}\right)+S_{f_{1}} S_{f_{2} \cdots f_{n}}-S_{f_{1} f_{2} \cdots f_{n}},
$$

the statement follows by induction.

If $\mathcal{F}$ is a subset of $L^{\infty}(\mathbb{D})$, then we write $\mathcal{I}(\mathcal{F})$ for the smallest closed subalgebra of $\mathcal{B}\left(\left(L_{a}^{2}\right)^{\perp}\right)$ containing $\left\{S_{f}: f \in \mathcal{F}\right\}$. The dual Toeplitz algebra is $\mathcal{I}\left(L^{\infty}(\mathbb{D})\right)$. Let $\mathcal{D}$ be the semicommutator ideal of the dual Toeplitz algebra $\mathcal{I}\left(L^{\infty}(\mathbb{D})\right)$. The following result states the existence of a symbol map from the dual Toeplitz algebra $\mathcal{I}\left(L^{\infty}(\mathbb{D})\right)$ to $L^{\infty}(\mathbb{D})$.

Theorem 8.3. There is a contractive $C^{*}$-homomorphism $\rho$ from the dual Toeplitz algebra $\mathcal{I}\left(L^{\infty}(\mathbb{D})\right)$ to $L^{\infty}(\mathbb{D})$ such that $\rho\left(S_{f}\right)=f$, for all $f \in L^{\infty}(\mathbb{D})$.

Proof. First we define $\rho$ on finite sums of finite products of dual Toeplitz operators. If $S=\sum_{i=1}^{n} S_{f_{i 1}} S_{f_{i 2}} \cdots S_{f_{i n_{i}}}$, we define $\rho(S)$ by

$$
\rho(S)=\sum_{i=1}^{n} f_{i 1} f_{i 2} \cdots f_{i n_{i}} .
$$

We must show that $\rho(S)$ is well-defined. Suppose that $S$ has another representation: $S=\sum_{i=1}^{m} S_{g_{i 1}} S_{g_{i 2}} \cdots S_{g_{i m_{i}}}$. Let

$$
F=\sum_{i=1}^{n} f_{i 1} f_{i 2} \cdots f_{i n_{i}} \text { and } G=\sum_{i=1}^{m} g_{i 1} g_{i 2} \cdots g_{i n_{i}} .
$$

We need only to show that $F(w)=G(w)$ a.e. on $\mathbb{D}$. By Proposition 8.2 both $S-S_{F}$ and $S-S_{G}$ are in the semicommutator ideal $\mathcal{D}$. Thus $S_{F}-S_{G}$ is in $\mathcal{D}$. By Lemma 8.1 we have

$$
\lim _{s \rightarrow 0^{+}}\left\|\left(S_{F}-S_{G}\right) u_{w, s}\right\|_{2}=0
$$

for a.e. $w \in \mathbb{D}$. On the other hand, Lemma 7.2 gives that

$$
|F(w)-G(w)|=\lim _{s \rightarrow 0^{+}}\left\|\left(S_{F}-S_{G}\right) u_{w, s}\right\|_{2} .
$$

Thus $F(w)=G(w)$ a.e. on $\mathbb{D}$, so that $\rho(S)$ is well-defined.

For each $S \in \mathcal{I}\left(L^{\infty}(\mathbb{D})\right)$ and a given positive integer $n$ there is a finite sum $F_{n}$ of finite products of dual Toeplitz operators such that

$$
\left\|S-F_{n}\right\|<1 / n \text {. }
$$


By the first part of the proof, $\rho\left(F_{n}\right)$ is well-defined. The sequence $\left(\rho\left(F_{n}\right)\right)$ in $L^{\infty}(\mathbb{D})$ is a Cauchy sequence, since

$$
\left\|\rho\left(F_{n}\right)-\rho\left(F_{m}\right)\right\|_{\infty} \leq\left\|F_{n}-F_{m}\right\| .
$$

We define $\rho(S)$ to be the limit of the Cauchy sequence $\left(\rho\left(F_{n}\right)\right)$ in $L^{\infty}(\mathbb{D})$. It is easily seen that $\rho(S)$ does not depend on the chosen sequence $\left(F_{n}\right)$.

The mapping $\rho$ is clearly linear, and it is easily seen that $\rho\left(S^{*}\right)=\overline{\rho(S)}$. To prove that $\rho$ is contractive it is sufficient to show that $\|\rho(S)\|_{\infty} \leq\|S\|$ if $S$ is a finite sum of finite products of dual Toeplitz operators. Writing $F=\rho(S)$, the operator $D=S-S_{F}$ is in the semicommutator ideal $\mathcal{D}$, so that by Lemma 8.1 $\left\|D u_{w, s}\right\|_{2} \rightarrow 0$ as $s \rightarrow 0^{+}$. Using Lemma 7.2 it follows that

$$
\|S\|=\left\|S_{F}+D\right\| \geq \lim _{s \rightarrow 0^{+}}\left\|\left(S_{F}+D\right) u_{w, s}\right\|_{2}=|F(w)|,
$$

for a.e. $w \in \mathbb{D}$, proving that indeed $\|\rho(S)\|_{\infty}=\|F\|_{\infty} \leq\|S\|$.

To prove that $\rho$ is a $C^{*}$-algebra homomorphism it suffices to prove that $\rho(S T)=$ $\rho(S) \rho(T)$, for operators $S$ and $T$ which are finite products of dual Toeplitz operators. Clearly it will be sufficient to show that

$$
\rho\left(S_{f_{1}} \cdots S_{f_{n}}\right)=\rho\left(S_{f_{1}}\right) \cdots \rho\left(S_{f_{n}}\right)
$$

for $f_{1}, \cdots, f_{n} \in L^{\infty}(\mathbb{D})$. This follows immediately from Proposition 8.2

We call $\rho$ the symbol map on the dual Toeplitz algebra $\mathcal{I}\left(L^{\infty}(\mathbb{D})\right)$. Define the mapping $\xi: L^{\infty}(\mathbb{D}) \longrightarrow \mathcal{L}\left(\left(L_{a}^{2}\right)^{\perp}\right)$ by $\xi(f)=S_{f}$, for $f \in L^{\infty}(\mathbb{D})$.

Theorem 8.4. If $\mathcal{D}$ is the semicommutator ideal in the dual Toeplitz algebra $\mathcal{I}\left(L^{\infty}(\mathbb{D})\right)$, then the mapping $\bar{\xi}$ induced from $L^{\infty}(\mathbb{D})$ to $\mathcal{I}\left(L^{\infty}(\mathbb{D})\right) / \mathcal{D}$ by $\xi$ is a *-isometric isomorphism. Thus there is a short exact sequence

$$
(0) \longrightarrow \mathcal{D} \longrightarrow \mathcal{I}\left(L^{\infty}(\mathbb{D})\right) \stackrel{\rho}{\longrightarrow} L^{\infty}(\mathbb{D}) \longrightarrow(0)
$$

for which $\xi$ is an isometric cross section.

Proof. The mapping $\bar{\xi}$ is obviously linear and contractive. To show that $\bar{\xi}$ is multiplicative, observe that for functions $f$ and $g$ in $L^{\infty}(\mathbb{D})$,

$$
\xi(f) \xi(g)-\xi(f g)=S_{f} S_{g}-S_{f g}
$$

is in the semicommutator $\mathcal{D}$. Thus $\bar{\xi}$ is multiplicative on $L^{\infty}(\mathbb{D})$.

To complete the proof we show that $\left\|S_{f}+K\right\| \geq\left\|S_{f}\right\|$ for $\varphi \in L^{\infty}(\mathbb{D})$ and $K$ in $\mathcal{D}$, and hence $\bar{\xi}$ is an isometry. Note that $\left\|S_{f}\right\|=\|f\|_{\infty}$. So it suffices to show that $\left\|S_{f}+K\right\| \geq\|f\|_{\infty}$. Since $K$ is in the semicommutator, by Theorem 8.3 we have

$$
\lim _{s \rightarrow 0^{+}}\left\|K u_{w, s}\right\|=0
$$

for all $w \in \mathbb{D}$. By Lemma 7.2 we also have that

$$
\lim _{s \rightarrow 0^{+}}\left\|S_{f} u_{w, s}\right\|=|f(w)|,
$$

for a.e. $w \in \mathbb{D}$. Thus

$$
\left\|S_{f}+K\right\| \geq \lim _{s \rightarrow 0^{+}}\left\|\left(S_{f}+K\right) u_{w, s}\right\|=|f(w)|
$$

for a.e. $w \in \mathbb{D}$. So this gives that $\left\|S_{f}+K\right\| \geq\|f\|_{\infty}$, which completes the proof.

Theorem 8.5. The semicommutator ideal in the dual Toeplitz algebra $\mathcal{I}\left(L^{\infty}(\mathbb{D})\right)$ contains the ideal $\mathcal{K}$ of compact operators on $\left(L_{a}^{2}\right)^{\perp}$. 
Proof. Let $\mathcal{D}$ denote the semicommutator ideal of $\mathcal{I}\left(L^{\infty}(\mathbb{D})\right)$. First we show that $\mathcal{D}$ contains the rank one operator $\bar{z} \otimes \bar{z}$.

As a special case of (2.5) we have

$$
\begin{aligned}
\bar{z} \otimes \bar{z} & =\left(H_{\bar{z}} 1\right) \otimes\left(H_{\bar{z}} 1\right)=H_{\bar{z}}(1 \otimes 1) H_{\bar{z}}^{*}=\mathcal{S}_{0}^{2}\left(H_{\bar{z}} H_{\bar{z}}^{*}\right) \\
& =H_{\bar{z}} H_{\bar{z}}^{*}-2 S_{z} H_{\bar{z}} H_{\bar{z}}^{*} S_{\bar{z}}+S_{z}^{2} H_{\bar{z}} H_{\bar{z}}^{*} S_{\bar{z}}^{2} .
\end{aligned}
$$

By (2.1),

$$
H_{\bar{z}} H_{\bar{z}}^{*}=S_{\bar{z} z}-S_{\bar{z}} S_{z} \in \mathcal{D} \text {. }
$$

Thus $\bar{z} \otimes \bar{z}$ is in $\mathcal{D}$.

Next we will show that the set $\mathcal{D}$ is irreducible in $\mathcal{B}\left(\left(L_{a}^{2}\right)^{\perp}\right)$. Let $\mathcal{N}$ be a closed linear subspace of $\mathcal{B}\left(\left(L_{a}^{2}\right)^{\perp}\right)$ which is reducing for $\mathcal{D}$. We have to show that $\mathcal{N}=\mathcal{B}\left(\left(L_{a}^{2}\right)^{\perp}\right)$. We will first prove the following claim.

Claim. The function $\bar{z}$ is in $\mathcal{N}$.

Since $\mathcal{N}$ is nonzero, it contains a nonzero function $\varphi$. Since the linear combinations of the functions $z^{m} \bar{z}^{n}$ are dense in $L^{2}(\mathbb{D}, d A)$ and $\varphi$ is not the zero function, $\varphi$ cannot be orthogonal to all $z^{m} \bar{z}^{n}$, and thus there exist integers $m, n \geq 0$ such that $\left\langle\varphi, z^{m} \bar{z}^{n}\right\rangle \neq 0$. Since $\varphi \in\left(L_{a}^{2}\right)^{\perp}$ is orthogonal to the function $z^{m}$, we must have $n>0$. Note that

$$
\left\langle\varphi, z^{m} \bar{z}^{n}\right\rangle \bar{z}=\left\langle\bar{z}^{m} z^{n-1} \varphi, \bar{z}\right\rangle \bar{z}=\left\langle S_{\bar{z}^{m} z^{n-1}} \varphi, \bar{z}\right\rangle \bar{z}=(\bar{z} \otimes \bar{z}) S_{\bar{z}^{m} z^{n-1}} \varphi .
$$

By the first part of the proof, $\bar{z} \otimes \bar{z} \in \mathcal{D}$. Since $\mathcal{D}$ is an ideal, $(\bar{z} \otimes \bar{z}) S_{\bar{z}^{m} z^{n-1}} \in \mathcal{D}$. Because $\mathcal{N}$ is reducing for every operator in $\mathcal{D}$, we have $\left\langle\varphi, z^{m} \bar{z}^{n}\right\rangle \bar{z} \in \mathcal{N}$. Because $\left\langle\varphi, z^{m} \bar{z}^{n}\right\rangle \neq 0$, we conclude that $\bar{z} \in \mathcal{N}$, and our claim is proved.

Now let $\psi$ be a function in $\left(L_{a}^{2}\right)^{\perp}$ which is orthogonal to $\mathcal{N}$. If $n>0$ and $m \geq 0$ are integers, then $(\bar{z} \otimes \bar{z}) S_{\bar{z}^{m} z^{n-1}} \in \mathcal{D}$, and since $\mathcal{N}$ is reducing for $\mathcal{D}$ it follows that

$$
\left\langle\psi, z^{m} \bar{z}^{n}\right\rangle \bar{z}=(\bar{z} \otimes \bar{z}) S_{\bar{z}^{m} z^{n-1}} \psi
$$

is orthogonal to $\mathcal{N}$. Because $\bar{z} \in \mathcal{N}$ we must have $\left\langle\psi, z^{m} \bar{z}^{n}\right\rangle=0$. Note that this is also true if $n=0$, since $\psi \in\left(L_{a}^{2}\right)^{\perp}$. So $\psi$ is orthogonal to all functions $z^{m} \bar{z}^{n}$, where $n \geq 0$ and $m \geq 0$ are integers. We conclude that $\psi=0$ a.e. on $\mathbb{D}$, and hence $\mathcal{N}$ equals $\left(L_{a}^{2}\right)^{\perp}$. This completes the proof that $\mathcal{D}$ is irreducible.

Note that $\mathcal{D}$ contains the nonzero compact operator $\bar{z} \otimes \bar{z}=H_{\bar{z}}(1 \otimes 1) H_{\bar{z}}^{*}$. By Theorem 5.39 of [9], $\mathcal{D}$ contains the ideal $\mathcal{K}$ of all compact operators on $\left(L_{a}^{2}\right)^{\perp}$.

Theorem 8.6. The $C^{*}$-algebra $\mathcal{I}(C(\overline{\mathbb{D}}))$ contains the ideal $\mathcal{K}$ of compact operators on $\left(L_{a}^{2}\right)^{\perp}$ as its semicommutator ideal, and the sequence

$$
(0) \rightarrow \mathcal{K} \rightarrow \mathcal{I}(C(\overline{\mathbb{D}})) \rightarrow C(\overline{\mathbb{D}}) \rightarrow(0)
$$

is short exact; that is, the quotient algebra $\mathcal{I}(C(\overline{\mathbb{D}})) / \mathcal{K}$ is $*$-isometrically isomorphic to $C(\overline{\mathbb{D}})$.

Proof. Write $\mathcal{S}$ to denote the semicommutator ideal in the dual Toeplitz algebra $\mathcal{I}(C(\overline{\mathbb{D}}))$. By the proof of the previous theorem, $\mathcal{K}$ is contained in $\mathcal{S}$. For two continuous functions $f$ and $g$ on $\overline{\mathbb{D}}$, by (2.1) the semicommutator

$$
S_{f g}-S_{f} S_{g}=H_{f} H_{\bar{g}}^{*}
$$


is compact. Since $\mathcal{S}$ is generated by semicommutators of dual Toeplitz operators with symbols in $C(\overline{\mathbb{D}})$, it follows that $\mathcal{S}$ is contained in $\mathcal{K}$. Hence $\mathcal{K}$ equals the semicommutator ideal $\mathcal{S}$. This completes the proof.

In Section 3 we have shown that the product of two dual Toeplitz operators cannot be zero unless one of the dual Toeplitz operators is the zero operator. The symbol mapping can be used to obtain generalizations of this result. In fact, we can obtain a necessary condition for the product of dual Toeplitz operators to be compact. We have the following generalization of Theorem 7.5

Theorem 8.7. Let $f_{1}, \ldots, f_{n} \in L^{\infty}(\mathbb{D})$. If the product $S_{f_{1}} S_{f_{2}} \cdots S_{f_{n}}$ is compact, then $f_{1}(w) f_{2}(w) \ldots f_{n}(w)=0$ for almost all $w$ in $\mathbb{D}$.

Proof. If $S_{f_{1}} S_{f_{2}} \cdots S_{f_{n}}$ is compact, then, by Theorem $8.5 S_{f_{1}} S_{f_{2}} \cdots S_{f_{n}}$ is in the semicommutator ideal $\mathcal{D}$. Using Proposition 8.2 we see that $S_{f_{1} f_{2} \cdots f_{n}}$ is in $\mathcal{D}$. It follows that $\rho\left(S_{f_{1} f_{2} \cdots f_{n}}\right)=0$, hence $f_{1} f_{2} \cdots f_{n}=0$ a.e. on $\mathbb{D}$.

The following result shows that a finite product of dual Toeplitz operators with bounded harmonic symbols cannot be zero unless one of the dual Toeplitz operators is zero. Whether or not the analogous statement is true for Toeplitz operators on the Hardy space is still an open problem.

Corollary 8.8. Let $f_{1}, \ldots, f_{n}$ be bounded harmonic functions on $\mathbb{D}$. The following statements are equivalent:

(i) $S_{f_{1}} S_{f_{2}} \cdots S_{f_{n}}$ is compact.

(ii) $S_{f_{1}} S_{f_{2}} \cdots S_{f_{n}}=0$.

(iii) One of the $f_{i}$ must be zero on $\mathbb{D}$.

\section{Spectral Properties of DUal Toeplitz operators}

In this section we discuss the spectrum and essential spectrum of dual Toeplitz operators.

Proposition 9.1. Let $f$ be a function in $L^{\infty}(\mathbb{D})$. If $S_{f}$ is invertible, then $f$ is invertible in $L^{\infty}(\mathbb{D})$.

Proof. Assume that for some $\delta>0$ we have $\left\|S_{f} u\right\|_{2} \geq \delta$, for all $u \in\left(L_{a}^{2}\right)^{\perp}$ with $\|u\|_{2}=1$. By Lemma 7.2, for a.e. $w \in \mathbb{D}$ we have

$$
|f(w)|=\lim _{s \rightarrow 0^{+}}\left\|S_{f} u_{w, s}\right\|_{2} \geq \delta .
$$

This completes the proof.

If $f$ is a measurable function on $\mathbb{D}$, then the essential range $\mathcal{R}(f)$ of $f$ is the set of all $\lambda$ in $\mathbb{C}$ for which $\{z \in \mathbb{D}:|f(z)-\lambda|<\epsilon\}$ has positive measure for every $\epsilon>0$. We have the following spectral inclusion theorem, completely analogous to the spectral inclusion theorem of Hartman and Wintner for Toeplitz operators on the Hardy space (see Corollary 7.7 in [9]):

Theorem 9.2. If $f$ is in $L^{\infty}(\mathbb{D})$, then $\mathcal{R}(f) \subset \sigma\left(S_{f}\right)$.

Proof. Since $S_{f}-\lambda=S_{f-\lambda}$ for $\lambda$ in $\mathbb{C}$, using Proposition 9.1 it follows that $\mathcal{R}(f) \subset$ $\sigma\left(S_{f}\right)$. 
Brown and Halmos [6] proved that the spectrum of a Toeplitz operator on the Hardy space is contained in the closed convex hull of the essential range of its symbol. For a subset $E$ of the complex plane we write $h(E)$ for the closed convex hull of $E$. The same argument as the proof of Corollary 7.19 in [9] shows that this is also true for dual Toeplitz operators.

Theorem 9.3. For every $f \in L^{\infty}(\mathbb{D})$ we have $\sigma\left(S_{f}\right) \subset h(\mathcal{R}(f))$.

A bounded operator $S$ on $\left(L_{a}^{2}\right)^{\perp}$ is Fredholm if and only if the operator $S+\mathcal{K}$ is invertible in the Calkin algebra $\mathcal{B}\left(\left(L_{a}^{2}\right)^{\perp}\right) / \mathcal{K}$. The following proposition states that a dual Toeplitz operator can only be Fredholm if its symbol is invertible.

Proposition 9.4. If $f$ is a function in $L^{\infty}(\mathbb{D})$ such that $S_{f}$ is a Fredholm operator, then $f$ is invertible in $L^{\infty}(\mathbb{D})$.

Proof. Let $\mathcal{K}$ denote the ideal of compact operators on $\left(L_{a}^{2}\right)^{\perp}$ and let $\mathcal{D}$ denote the ideal generated by the semicommutators of all bounded dual Toeplitz operators. If $S_{f}$ is Fredholm, then $S_{f}+\mathcal{K}$ is invertible in the Calkin algebra $\mathcal{B}\left(L^{\infty}(\mathbb{D})\right) / \mathcal{K}$. Since $\mathcal{I}\left(L^{\infty}(\mathbb{D})\right) / \mathcal{K}$ is a closed self-adjoint subalgebra of $\mathcal{B}\left(L^{\infty}(\mathbb{D})\right) / \mathcal{K}$, it follows from Theorem 4.28 of [9] that $S_{f}+\mathcal{K}$ is invertible in $\mathcal{I}\left(L^{\infty}(\mathbb{D})\right) / \mathcal{K}$. By Theorem 8.5] $\mathcal{K} \subset \mathcal{D}$, so $S_{f}+\mathcal{D}$ is invertible in the algebra $\mathcal{I}\left(L^{\infty}(\mathbb{D})\right) / \mathcal{D}$. It follows that $f=\rho\left(S_{f}\right)$ is invertible in $L^{\infty}(\mathbb{D})$.

The essential spectrum of a bounded linear operator $S$ on $\left(L_{a}^{2}\right)^{\perp}$, denoted by $\sigma_{e}(S)$, is the spectrum of $S+\mathcal{K}$ in the Calkin algebra $\mathcal{B}\left(\left(L_{a}^{2}\right)^{\perp}\right) / \mathcal{K}$. We have the following inclusion theorem for the essential spectrum of a dual Toeplitz operator:

Theorem 9.5. If $f$ is in $L^{\infty}(\mathbb{D})$, then $\mathcal{R}(f) \subset \sigma_{e}\left(S_{f}\right)$.

Proof. Since $S_{f}-\lambda=S_{f-\lambda}$ for $\lambda$ in $\mathbb{C}$, using Proposition 9.4 it follows that $\mathcal{R}(f) \subset$ $\sigma_{e}\left(S_{f}\right)$.

Theorem 9.6. If $f$ is in $L^{\infty}(\mathbb{D})$ and is such that both Hankel operators $H_{f}$ and $H_{\bar{f}}$ are compact, then $\sigma_{e}\left(S_{f}\right)=\mathcal{R}(f)$.

Proof. By the previous theorem it suffices to prove that $\sigma_{e}\left(S_{f}\right) \subset \mathcal{R}(f)$. If $\lambda \in$ $\mathbb{C} \backslash \mathcal{R}(f)$, then for some $\epsilon>0$ we have $|f(z)-\lambda| \geq \epsilon$, for a.e. $z$ in $\mathbb{D}$. Then $g=1 /(f-\lambda)$ is in $L^{\infty}(\mathbb{D})$. By $(2.1)$

$$
S_{f-\lambda} S_{g}=I-H_{f} H_{\bar{g}}^{*} \text { and } S_{g} S_{f-\lambda}=I-H_{g} H_{\bar{f}}^{*} .
$$

Since both $H_{f} H_{\bar{g}}^{*}$ and $H_{g} H_{\bar{f}}^{*}$ are compact, $S_{f-\lambda}+\mathcal{K}$ is invertible in the Calkin algebra, so that $\lambda \in \mathbb{C} \backslash \sigma_{e}\left(S_{f}\right)$.

Widom 23] proved that the spectrum of a Toeplitz operator on the Hardy space is connected, and Douglas [9] proved that also the essential spectrum is connected. These statements are no longer true for dual Toeplitz operators.

Proposition 9.7. If $f$ is a non-constant real-valued simple measurable function on $\mathbb{D}$ for which $H_{f}$ is compact, then both $\sigma\left(S_{f}\right)$ and $\sigma_{e}\left(S_{f}\right)$ are disconnected.

Proof. By Theorem 9.6, $\sigma_{e}\left(S_{f}\right)=\mathcal{R}(f)$, which is a finite set consisting of more than one point. Since $S_{f}$ is self-adjoint, eigenvectors corresponding to distinct eigenvalues are orthogonal. Because $\left(L_{a}^{2}\right)^{\perp}$ is a separable Hilbert space, the operator $S_{f}$ has at most countable point-spectrum $\sigma_{p}\left(S_{f}\right)$. Since $\sigma\left(S_{f}\right) \backslash \sigma_{e}\left(S_{f}\right) \subset \sigma_{p}\left(S_{f}\right)$, the spectrum $\sigma\left(S_{f}\right)$ is at most countable. Because $\sigma\left(S_{f}\right)$ contains $\mathcal{R}(f)$, the set $\sigma\left(S_{f}\right)$ must be disconnected. 
The following class of examples will show that modulo countable sets the spectrum of a dual Toeplitz operator can be any compact subset of the complex plane.

Example 9.8. Let $\left(r_{k}\right)$ be a strictly increasing sequence in the interval $[0,1)$ with $r_{0}=0$, and let $\left(w_{k}\right)$ be a bounded sequence of complex numbers. Consider the function $f: \mathbb{D} \rightarrow \mathbb{C}$ defined by

$$
f(z)=w_{k} \quad \text { for } \quad r_{k-1} \leq|z|<r_{k}, \quad k=1,2, \cdots .
$$

Put $W=\left\{w_{1}, w_{2}, \cdots\right\}$. Note that $\bar{W}=\mathcal{R}(f) \subset \sigma_{e}\left(S_{f}\right)$. Suppose that $\lambda$ is an eigenvalue of $S_{f}$ not in $\bar{W}$. Then there exists a nonzero $h$ in $\left(L_{a}^{2}\right)^{\perp}$ such that $S_{f} h=\lambda h$. Then the function $g=(f-\lambda) h=f h-S_{f} h=P(f h)$ is in $L_{a}^{2}$. If $r_{k-1} \leq|z|<r_{k}$, then $f(z)=w_{k}$; thus $h(z)=g(z) /\left(w_{k}-\lambda\right)$. If $g(z)=\sum_{n=0}^{\infty} a_{n} z^{n}$, then for each $n$ we have

$$
\begin{aligned}
0 & =\left\langle h, z^{n}\right\rangle=\sum_{k=1}^{\infty} \int_{r_{k-1} \leq|z|<r_{k}} h(z) \bar{z}^{n} d A(z) \\
& =\sum_{k=1}^{\infty} \int_{r_{k-1} \leq|z|<r_{k}} \frac{g(z)}{w_{k}-\lambda} \bar{z}^{n} d A(z) \\
& =\sum_{k=1}^{\infty} \frac{a_{n}}{w_{k}-\lambda} \int_{r_{k-1} \leq|z|<r_{k}}|z|^{2 n} d A(z),
\end{aligned}
$$

hence $\lambda$ satisfies the equations

$$
\frac{a_{n}}{n+1} \sum_{k=1}^{\infty} \frac{r_{k}^{2 n+2}-r_{k-1}^{2 n+2}}{w_{k}-\lambda}=0
$$

for all $n=0,1, \cdots$. Since $g$ is not the zero function, $a_{n} \neq 0$ for some $n$, and consequently

$$
\sum_{k=1}^{\infty} \frac{r_{k}^{2 n+2}-r_{k-1}^{2 n+2}}{w_{k}-\lambda}=0 .
$$

For each non-negative integer $n$ the function

$$
\varphi_{n}(z)=\sum_{k=1}^{\infty} \frac{r_{k}^{2 n+2}-r_{k-1}^{2 n+2}}{w_{k}-z}
$$

is analytic on $\mathbb{C} \backslash \bar{W}$ : if $z$ is not in $\bar{W}$, then $\left|z-w_{k}\right| \geq \delta>0$, for all $k=1,2, \cdots$, and thus

$$
\frac{r_{k}^{2 n+2}-r_{k-1}^{2 n+2}}{\left|w_{k}-z\right|} \leq \delta^{-1}\left(r_{k}^{2 n+2}-r_{k-1}^{2 n+2}\right)
$$

Hence

$$
\begin{aligned}
\sum_{k=1}^{\infty} \frac{r_{k}^{2 n+2}-r_{k-1}^{2 n+2}}{\left|w_{k}-z\right|} & \leq \delta^{-1} \sum_{k=1}^{\infty}\left(r_{k}^{2 n+2}-r_{k-1}^{2 n+2}\right) \\
& =\delta^{-1} r_{\infty}^{2 n+2} \leq \delta^{-1}<\infty
\end{aligned}
$$

where $r_{\infty}=\lim _{k \rightarrow \infty} r_{k}$.

Thus the point-spectrum of $S_{f}$ is contained in the union of $\bar{W}$ with the zero sets $Z\left(\varphi_{n}\right)$ of the functions $\varphi_{n}$. The equations determining the eigenvalues of $S_{f}^{*}=S_{\bar{f}}$ not in the closure of the $\bar{w}_{k}$ 's are identical to the above equations (just the complex 
conjugates of them). Using the notation $E^{*}=\{\bar{z}: z \in E\}$, for a set $E$ of complex numbers, for any bounded operator $T$ we have

$$
\sigma(T) \backslash \sigma_{e}(T) \subset \sigma_{p}(T) \cup \sigma_{p}\left(T^{*}\right)^{*} .
$$

We conclude that

$$
\sigma\left(S_{f}\right) \subset \sigma_{e}\left(S_{f}\right) \cup \bigcup_{n=0}^{\infty} Z\left(\varphi_{n}\right) .
$$

If $f$ is furthermore constant near the circle (i.e., $r_{\infty}<1$ ), then both operators $H_{f}$ and $H_{\bar{f}}$ are compact; thus $\sigma_{e}\left(S_{f}\right)=\mathcal{R}(f)=\bar{W}$, and we obtain

$$
\bar{W} \subset \sigma\left(S_{f}\right) \subset \bar{W} \cup \bigcup_{n=0}^{\infty} Z\left(\varphi_{n}\right) .
$$

So, if $W$ is an infinite set with a finite number of accumulation points, then $\sigma\left(S_{f}\right)$ is countably infinite and thus disconnected. The set $Z=\bigcup_{n=0}^{\infty} Z\left(\varphi_{n}\right)$ is countable; $\bar{W}$ can be any compact subset of the complex plane, so the above inclusions show:

For every compact subset $K$ of the complex plane there exists a bounded measurable function $f$ such that $\sigma\left(S_{f}\right)=K \cup N$, where $N$ is a countable set.

Note that it is not necessary for $r_{\infty}$ to be strictly less than 1 in order for $H_{f}$ and $H_{\bar{f}}$ to be compact. If we take

$$
r_{2 k}=1-\frac{1}{2 k+1}, \quad r_{2 k+1}=1-\frac{1}{2 k+1+2^{-2 k-1}},
$$

and $w_{k}=\left(1+(-1)^{k}\right) / 2$, then in [8] it was shown that the Hankel operator $H_{f}=H_{\bar{f}}$ is Hilbert-Schmidt.

In the special case that $f$ is the function

$$
f(z)= \begin{cases}w_{1} & \text { for }|z|<r \\ w_{2} & \text { for } r \leq|z|<1\end{cases}
$$

equations (9.9) are equivalent to

$$
a_{n}\left\{\frac{r^{2 n+2}}{w_{1}-\lambda}+\frac{1-r^{2 n+2}}{w_{2}-\lambda}\right\}=0
$$

for $n=0,1, \cdots$. If $a_{n} \neq 0$, then (9.10) has solution $\lambda=w_{1}+\left(w_{2}-w_{1}\right) r^{2 n+2}$. If $\lambda=w_{1}+\left(w_{2}-w_{1}\right) r^{2 n+2}$ and $h$ is the function on $\mathbb{D}$ defined by

$$
h(z)= \begin{cases}\frac{z^{n}}{w_{1}-\lambda} & \text { if }|z|<r, \\ \frac{z^{n}}{w_{2}-\lambda} & \text { if } r \leq|z|<1,\end{cases}
$$

then $(f-\lambda) h=z^{n}$, and thus $S_{f-\lambda} h=0$. So $\lambda$ is indeed an eigenvalue. Note that (9.10) shows that the power series coefficients of $g$ other than $a_{n}$ must be 0 . This easily implies that $S_{f-\lambda}$ has one-dimensional kernel. We conclude that in this case

$$
\sigma\left(S_{f}\right)=\left\{w_{1}, w_{2}\right\} \cup\left\{w_{1}+\left(w_{2}-w_{1}\right) r^{2 n+2}: n=0,1,2, \cdots\right\} .
$$

Observe that this set does belong to the closed convex hull of $w_{1}$ and $w_{2}$.

Thus there are bounded measurable functions $f$ on $\mathbb{D}$ for which both $\operatorname{ker}\left(S_{f}\right)$ and $\operatorname{ker}\left(S_{f}^{*}\right)$ are non-trivial, in constrast with Coburn's result for Toeplitz operators on the Hardy space (see Proposition 7.24 in [9]). 
The above example shows a difference with Toeplitz operators on the Bergman space. If $f$ is the characteristic function of a countable union of annuli in the unit disk centered at 0 , then Lark [14] has shown that $\sigma_{e}\left(T_{f}\right)$ is connected. However, as is the case for dual Toeplitz operators, $\sigma\left(T_{f}\right)$ may be disconnected. If $g$ is a function as described in Section 6 of [20] such that $T_{g}$ is compact, then $f=\frac{1}{2}(1+g)$ is a characteristic function of a countable union of annuli in the unit disk centered at 0 , and $\sigma\left(T_{f}\right)=\left\{\frac{1}{2}+\lambda_{n}: n=0,1,2, \cdots\right\}$, where the $\lambda_{n}$ are positive numbers converging to 0 . Clearly this set is disconnected.

In contrast to Proposition 9.7 we have the following result.

Proposition 9.11. If $f$ is a continuous real-valued function on $\mathbb{D}$, then $\sigma\left(S_{f}\right)=$ $\sigma_{e}\left(S_{f}\right)$ is connected.

Proof. Since $f$ is continuous and real-valued, $\mathcal{R}(f)=\overline{f(\mathbb{D})}$ is an interval. Combining Theorem 9.5 and Theorem 9.3 we see that $\sigma_{e}\left(S_{f}\right)=\sigma\left(S_{f}\right)=\overline{f(\mathbb{D})}$.

By the above proposition the spectrum of a dual Toeplitz operator with bounded real-valued harmonic symbol is connected. We do not know whether this is also true for dual Toeplitz operators with bounded complex-valued harmonic symbols. For bounded analytic and co-analytic symbols we have the following result.

Theorem 9.12. If $f$ is a bounded analytic or co-analytic function on $\mathbb{D}$, then $\sigma\left(S_{f}\right)=\sigma_{e}\left(S_{f}\right)=\overline{f(\mathbb{D})}$.

Proof. Since $S_{f}^{*}=S_{\bar{f}}$, it suffices to consider the case that $f$ is co-analytic on $\mathbb{D}$. If $f$ is conjugate analytic, then $L_{a}^{2}$ is invariant under the multiplication operator $M_{\bar{f}}$, so $\left(L_{a}^{2}\right)^{\perp}$ is invariant under $M_{f}$. Thus $S_{f}$ is the restriction of $M_{f}$ to $\left(L_{a}^{2}\right)^{\perp}$. If a complex number $\lambda$ is not in $\overline{f(\mathbb{D})}$, then $S_{f-\lambda}$ has inverse $S_{1 /(f-\lambda)}$. Hence $\sigma\left(S_{f}\right) \subset \overline{f(\mathbb{D})}$. By Theorem $9.5 f(\mathbb{D}) \subset \sigma_{e}\left(S_{f}\right)$, and the conclusion follows.

\section{REFERENCES}

1. P. Ahern and Ž. Čučković, Products of Toeplitz Operators on the Bergman Space, Illinois J. Math. 45 (2001), 113-121.

2. S. Axler, Bergman spaces and their operators, Surveys of Some Recent Results in Operator Theory, volume I, edited by J.B. Conway and B.B. Morrel, Pitman Research Notes in Mathematics, 1986, pp. 1-50. MR 90b:47048

3. S. Axler and Ž. Cučković, Commuting Toeplitz operators with harmonic symbols, Integral Equations Operator Theory 14 (1991), 1-12. MR 92f:47018

4. S. Axler and D. Zheng, The Berezin transform on the Toeplitz algebra, Studia Math. 127 (1998), 113-136. MR 98m:47030

5. S. Axler and D. Zheng, Compact operators via the Berezin transform, Indiana Univ. Math. J. 47 (1998), 387-400. MR 99i:47045

6. A. Brown and P.R. Halmos, Algebraic Properties of Toeplitz Operators, J. Reine Angew. Math. 213 (1963/1964), 89-102. MR 28:3350

7. J. Bunce, The joint spectrum of commuting non-normal operators, Proc. Amer. Math. Soc. 29 (1971), 499-504. MR 44:832

8. J.A. Cima, K. Stroethoff, and K. Yale, Bourgain algebras on the unit disk, Pacific J. Math. 160 (1993), no.1, 27-41. MR 94i:96065

9. R. Douglas, Banach algebra techniques in operator theory, Academic Press, San Diego, 1972. MR 50:14335

10. J.B. Garnett, Bounded Analytic Functions, Academic Press, Orlando, 1981. MR 83g:30037

11. P. Gorkin and D. Zheng, Essentially Commuting Toeplitz Operators, Pacific J. Math. 190 (1999), 87-109. MR 2000j:47054

12. P. Hartman and A. Wintner, The spectra of Toeplitz's matrices, Amer. J. Math. 76 (1954), 867-882. MR 17:499a 
13. K. Hoffman, Bounded analytic functions and Gleason parts, Ann. of Math. 86 (1967), 74-111. MR 35:5945

14. J.W. Lark, Spectral theorems for a class of Toeplitz operators on the Bergman space, Houston J. Math. 12 (1986), no. 3, 397-404. MR 88i:47012

15. G. McDonald and C. Sundberg, Toeplitz operators on the disc, Indiana Univ. Math. J. 28 (1979), 595-611. MR 80h:47034

16. N.K. Nikol'skiı,, Treatise on the shift operator, Springer-Verlag, New York, 1985. MR 87i: 47042

17. W. Rudin, Real and Complex Analysis, 2nd ed., McGraw-Hill, New York, 1974. MR 49:8783

18. D. Sarason, Function theory on the unit circle, Virginia Poly. Inst. and State Univ., Blacksburg, Virginia, 1979. MR 80d:30035

19. K. Stroethoff, Essentially commuting Toeplitz operators with harmonic symbols, Canadian Math. J. 45 (1993), 1080-1093. MR 94h:47046

20. K. Stroethoff, The Berezin Transform and Operators on Spaces of Analytic Functions, Linear Operators, edited by J. Zemánek, Banach Center Publications, Vol. 38, Polish Academy of Sciences, Warsaw (1997), 361-380. MR 98g:47025

21. K. Stroethoff and D. Zheng, Toeplitz and Hankel operators on Bergman spaces, Trans. Amer. Math. Soc. 329 (1992), no. 2, 773-794. MR 92e:47044

22. K. Stroethoff and D. Zheng, Products of Hankel and Toeplitz Operators on the Bergman space, J. Functional Analysis 169 (1999), 289-313. MR 2000i:47053

23. H. Widom, On the spectrum of a Toeplitz operator, Pacific J. Math. 14 (1964), 365-375. MR 29:476

24. K. Zhu, Operator Theory on Function Spaces, Marcel Dekker, New York, 1990. MR 92c:47031

Department of Mathematical Sciences, University of Montana, Missoula, Montana 59812

E-mail address: ma_kms@selway.umt.edu

Mathematics Department, Vanderbilt University, Nashville, Tennessee 37240

E-mail address: zheng@math.vanderbilt.edu 